\title{
Intracranial Dural Arteriovenous Fistulas With Brainstem Engorgement: An Under-Recognized Entity in Diagnosis and Treatment
}

\author{
Kun $\mathrm{Hou}^{1+}$, Guichen $\mathrm{Li}^{2 \dagger}$, Lai Qu ${ }^{3}$, Hongping $\mathrm{Liu}^{2}$, Kan $\mathrm{Xu}{ }^{1 * \neq}$ and Jinlu Yu${ }^{1 * \neq}$ \\ ${ }^{1}$ Department of Neurosurgery, The First Hospital of Jilin University, Changchun, China, ${ }^{2}$ Department of Neurology, The First \\ Hospital of Jilin University, Changchun, China, ${ }^{3}$ Department of Intensive Care Unit, The First Hospital of Jilin University, \\ Changchun, China
}

\section{OPEN ACCESS}

Edited by:

Atilla Ozcan Ozdemir,

Eskişehir Osmangazi University, Turkey

Reviewed by:

Ashish Kulhari,

JFK Medical Center, United States Waldo Rigoberto Guerrero, University of South Florida,

United States

${ }^{*}$ Correspondence:

Jinlu Yu

jlyu@jlu.edu.cn

Kan Xu

xukanjlu@163.com

these authors have contributed equally to this work and share first

authorship

¥These authors have contributed equally to this work

Specialty section:

This article was submitted to

Endovascular and Interventional

Neurology,

a section of the journal

Frontiers in Neurology

Received: 28 January 2020

Accepted: 28 August 2020

Published: 25 September 2020

Citation:

Hou K, Li G, Qu L, Liu H, Xu K and

Yu J (2020) Intracranial Dural Arteriovenous Fistulas With Brainstem Engorgement: An Under-Recognized Entity in Diagnosis and Treatment.

Front. Neurol. 11:526550

doi: $10.3389 /$ fneur.2020.526550
Background: In rare circumstances, patients with intracranial (dural arteriovenous fistulas) DAVFs could be complicated with brainstem engorgement, which might lead to delayed or false diagnosis and subsequent improper management.

Methods: On July 2th, 2019, a systematic search was conducted in the PubMed database for patients with intracranial DAVFs complicated with brainstem engorgement.

Results: Sixty-eight articles reporting of 86 patients were included for final analysis. The patients were aged from 20 to 76 years $(57.10 \pm 12.90, n=82)$. The female to male ratio was 0.68 (35:51). Thirty-three $(40.2 \%, 33 / 82)$ patients were initially misdiagnosed as other diseases. The specific location distributions were cranio-cervical junction, cavernous sinus, superior petrosal sinus, transverse and/or sigmoid sinus, tentorium, and other sites in 27 (32.5\%), 11 (13.2\%), 9 (10.8\%), 10 (12.0\%), 21 (25.3\%), and 5 (6.0\%) patients, respectively. The Cognard classification of DAVFs were II, III, IV, and V in $9(10.7 \%, 9 / 84), 1(1.2 \%, 1 / 84), 1$ (1.2\%, 1/84), and 73 (86.9\%, 73/84) patients. Eighteen $(22 \%, 18 / 82)$ patients were demonstrated to have stenosis or occlusion of the draining system distal to the fistula points. The mean follow-up period was 7.86 ( $n=74$, range 0-60 months) months. Fifty-four $(70.1 \%, 54 / 77)$ patients experienced a good recovery according to the mRS score.

Conclusions: Intracranial DAVFs complicated with brainstem engorgement are rare entities. Initial misdiagnosis and delayed definite diagnosis are common in the past three decades. The treatment outcome is still unsatisfactory at present. Early awareness of this rare entity and efficiently utilizing the up to date investigations are of utmost importance.

Keywords: dural arteriovenous fistula, brainstem engorgement, transarterial embolization, transvenous embolization, open surgery

\section{INTRODUCTION}

Dural arteriovenous fistula (DAVF) is a unique subtype of vascular malformations along the central nervous system, which is characterized by abnormal connections between meningeal/pial arteries and dural venous sinuses, meningeal veins, or cortical veins. The estimated detection rate was 0.29 per 100,000 persons per year according to a Japanese survey published in 
2016 (1). In rare circumstances, patients with intracranial DAVFs could be complicated with brainstem engorgement, which might lead to delayed or false diagnosis and subsequent improper management (2-4). An illustrate case of intracranial DAVF with brainstem engorgement was presented in Figure 1. As a result of its rarity in occurrence, large case series in a single center is extremely hard to be anticipated. In order to explore the epidemiological, clinical, imaging, and prognostic characteristics of this specific entity, we conducted a systematic review of the literature.

\section{METHODS}

On July 2th, 2019, a systematic search was conducted in the PubMed database for patients with intracranial DAVFs complicated with brainstem engorgement. Brainstem engorgement, brain stem engorgement, brainstem edema, brainstem oedema, brain stem edema, brain stem oedema, brainstem congestion, brain stem congestion, brainstem venous congestion, brain stem venous congestion, venous congestion of brain stem, venous congestion of brainstem, myelopathy, and
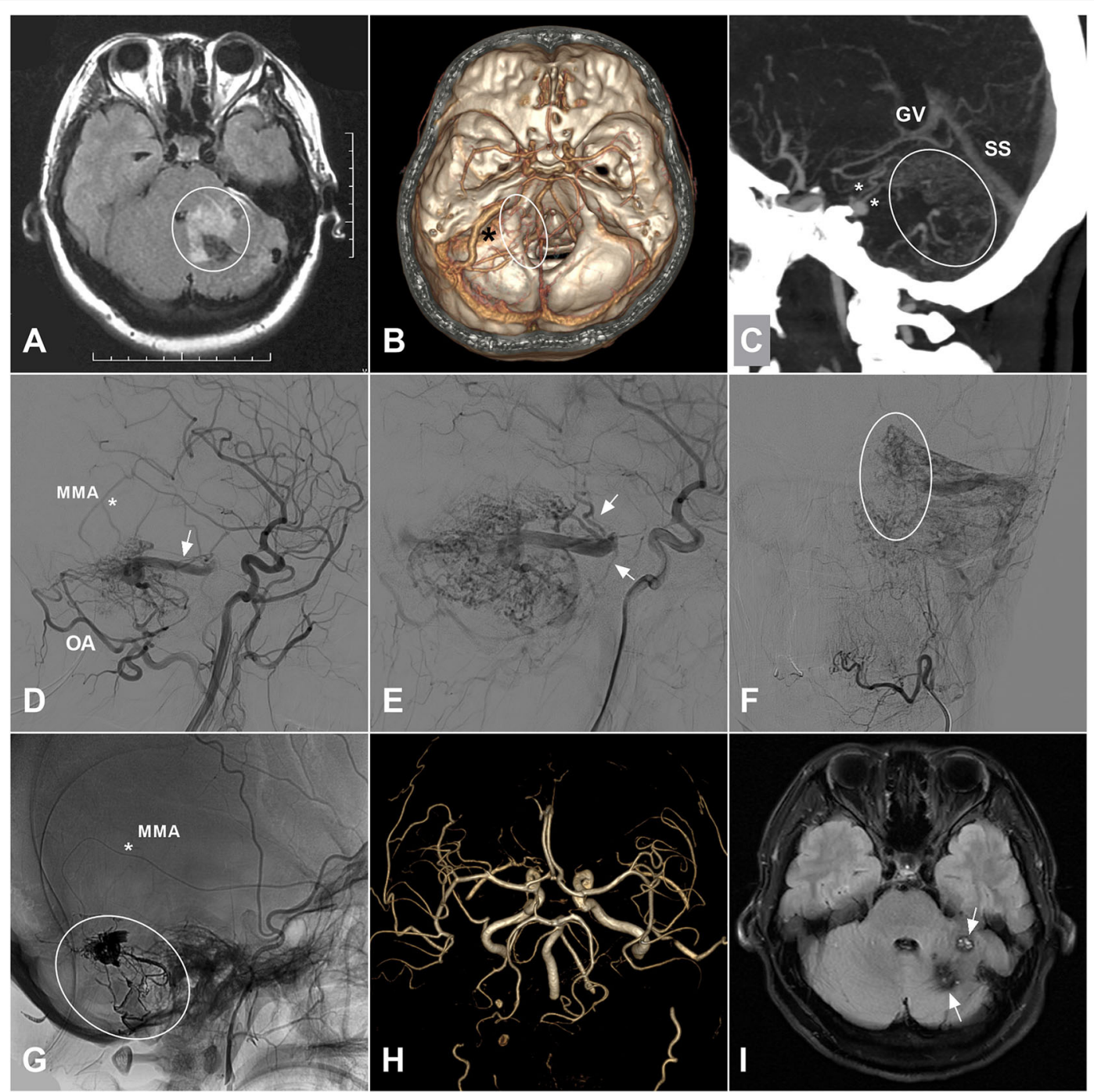

FIGURE 1 | (A) A 35-years-old female was admitted for 3-days history of headache and vomiting. MRI on FLAIR sequence reveals a hyperintense left cerebellar lesion (white circle) with the adjacent brainstem involvement. Besides, vascular flow voids are also noted at the posterior fossa. (B) CTA shows an abnormally enlarged vein (asterisk) draining from the cerebellar surface to the brainstem. And some enlarged veins (ellipse) around the brainstem are also noted. (C) MIP of CTA shows the enlarged draining veins in the cerebellum (ellipse) and around the brainstem (asterisks). (D) Angiogram of the left ECA in lateral view shows a DAVF supplied by the MMA (asterisk) and OA and drained to the deep veins via an enlarged superficial vein (arrow). (E) Angiogram in late arterial phase shows the deep veins (arrow) around the brainstem. (F) Angiogram of the left ECA in anteroposterior view shows enlarged veins in the left cerebellar hemisphere. The ellipse indicates the midline veins. (G) Unsubtracted angiogram shows the DAVF is embolized with Onyx (ellipse) via the MMA (asterisk). (H) Follow-up MRA 1 month postoperatively shows disappearance of the DAVF. (I) Follow-up MRI on FLAIR sequence shows remission of the brainstem and cerebellar edema and deposition of hemosiderin (arrow). CTA, computed tomography angiography; DAVF, dural arteriovenous fistula; ECA, external carotid artery; FLAIR, fluid attenuated inversion recovery; MIP, maximum intensity projection; MMA, middle meningeal artery; MRA, magnetic resonance angiography; MRI, magnetic resonance imaging; OA, occipital artery. 
dural arteriovenous fistula were used as key words in relevant combinations. Articles included were: (1) of which the full text could be obtained, or (2) sufficient data could be obtained from the abstract if the full text is inaccessible. Of note, studies reporting large case series were excluded from the final analysis if sufficient description of the individual clinical information was not provided. Manual searching of the reference lists of the identified articles were also performed for additional studies. We used modified Rankin Scale (mRS) for outcome assessment. An mRS score $\leq 3$ was defined as good recovery.

\section{RESULTS}

The PubMed search yielded 183 records. After a primary screening of the titles and abstracts, 97 records were excluded. After full text assessment of the 86 identified articles, 28 records were further excluded. We manually searched the reference lists of the remaining 58 articles. And 10 additional articles were identified. Finally, 68 articles reporting of 86 patients were included in the final analysis (Table 1) (2-69). The flow chart of searching strategy was presented in Figure 2. The patients were aged from 20 to 76 years $(57.10 \pm 12.90, n=82)$. The female to male ratio was 0.68 (35:51).

\section{Interval From Symptom Onset to Definite Diagnosis}

Of the 68 cases interval from symptom onset to definite diagnosis was provided, $15(22.1 \%, 15 / 68)$ patients were definitely diagnosed with intracranial DAVFs in the 1st month since symptom onset. Nineteen $(28.0 \%, 19 / 68)$ patients were definitely diagnosed between the 2 nd and 3 rd months. Sixteen $(23.5 \%$, $16 / 68)$ patients were between the fourth and 6th month. Six $(8.8 \%, 6 / 68)$ patients were between the seventh and twelfth month. Twelve $(17.6 \%, 12 / 68)$ patients were definitely diagnosed 1 year later from symptom onset. Thirty-three $(40.2 \%, 33 / 82)$ patients were initially misdiagnosed as other diseases.

\section{DAVFs Characteristics}

The intracranial location of DAVFs could be determined in 83 patients. The specific location distributions were anterior fossa, cranio-cervical junction, cavernous sinus, vein of Galen, occipital sinus, superior petrosal sinus, transverse/sigmoid sinus, torcular, and tentorium in 1 (1.2\%), 27 (32.5\%), 11 (13.2\%), 1 (1.2\%), 1 (1.2\%), 9 (10.8\%), 10 (12.0\%), 2 (2.4\%), and $21(25.3 \%)$ patients, respectively (Figure 3). The Cognard classification of DAVFs were II, III, IV, and V in $9(10.7 \%, 9 / 84), 1(1.2 \%$, $1 / 84), 1(1.2 \%, 1 / 84)$, and $73(86.9 \%, 73 / 84)$ patients (Figure 4). The feeding arteries were solely from the external carotid artery (ECA) in $32(38.6 \%, 32 / 83)$ patients, solely from the internal carotid artery (ICA) in $14(16.9 \%, 14 / 83)$ patients, solely from the vertebrobasilar artery (VBA) in $12(14.5 \%$, $12 / 83$ ) patients, conjointly from ECA and ICA in $18(21.7 \%$, $18 / 83)$ patients, conjointly from ECA and VBA in $5(6.0 \%, 5 / 83)$ patients, and conjointly from ECA, ICA, and VBA in $2(2.4 \%$, 2/83) patients.

\section{Findings on Imaging Modalities}

Eighteen $(22 \%, 18 / 82)$ patients were demonstrated to have stenosis or occlusion of the draining system distal to the fistula points during conventional angiography. The signals of the engorged brainstem were hypointense or normal on T1 weighted imaging (T1WI) of magnetic resonance imaging (MRI) in 15 $(65.2 \%, 15 / 23)$ and $8(34.8 \%, 8 / 23)$ patients, respectively. The engorged brainstem was enhanced on T1WI with different degrees in $37(72.5 \%, 37 / 51)$ patients after gadolinium contrast. The signal was hyperintense in all of the 82 patients T2 weighted imaging (T2WI) sequence was provided. And the signal was also hyperintense for all of the 25 patients who had undergone fluid attenuated inversion recovery (FLAIR) sequence. The signals on diffusion weighted imaging (DWI) were heterogeneous, hyperintense, hypointense, and normal in 1 (10\%), 3 (30\%), 1 $(10 \%)$, and $5(50 \%)$ patients, respectively. All of the six patients showed hyperintensity on apparent diffusion coefficient (ADC) map. Besides, abnormal vascular flow voids could be identified in $69(80.2 \%, 69 / 86)$ patients on MRI.

\section{Treatment and Outcome}

Forty-five $(53.6 \%, 45 / 84)$ patients were treated solely with transarterial embolization, of which $7(15.6 \%, 7 / 45)$ patients were incompletely embolized and $3(6.7 \%, 3 / 45)$ patients experienced recurrence in spite of previous complete obliteration. Eight $(9.5 \%, 8 / 84)$ patients underwent transvenous embolization, of which $1(12.5 \%, 1 / 8)$ patient was incompletely embolized. Twenty-two $(26.2 \%, 22 / 84)$ patients underwent open surgery, of which no recurrence was reported. One $(1.2 \%, 1 / 84)$ patient underwent one-session successful stereotactic radiosurgery. Eight $(9.5 \%, 8 / 84)$ patients were successfully treated conjointly with the endovascular and open surgical approaches. In general, the DAVFs were completely obliterated in $74(89.2 \%, 74 / 83)$ patients during one hospitalization. Six $(7.2 \%, 6 / 83)$ patients underwent retreatment. The mean follow-up period was 7.86 ( $n=74$, range $0-60$ months) months. Fifty-four $(70.1 \%, 54 / 77)$ patients experienced a good recovery according to the mRS score.

\section{DISCUSSION}

The pathophysiology of intracranial DAVFs is still enigmatic. Though a small proportion of the DAVFs are demonstrated to be secondary to trauma, craniotomy, infection, or dural venous thrombosis, a substantial number of them are idiopathic (70). Some authors believe that progressive stenosis or thrombosis of the dural venous sinus might be the underlying mechanism of DAVF formation $(61,70)$. In this review, $22 \%$ of the patients with brainstem engorgement were definitely recorded to have stenosis or occlusion of the draining system distal to the fistula points. The actual occurrence of stenosis or occlusion of the draining system might be higher, as some reports did not give a detailed description of the draining system. According to a study by Luo et al. 7 (77.8\%) of the nine patients with aggressive cavernous sinus DAVFs had inferior petrous sinus occlusion or stenosis, two patients (22.2\%) had compartment of inferior petrous sinus-cavernous sinus (77). Hence, progressive insufficient drainage (stenosis, occlusion, or compartment) of the 
TABLE 1 | Intracranial DAVFs complicated with brainstem engorgement.

\begin{tabular}{|c|c|c|c|c|c|c|c|c|c|c|c|c|c|c|c|c|c|c|c|c|}
\hline \multirow{2}{*}{\multicolumn{2}{|c|}{ No.Author/year }} & \multirow[t]{2}{*}{ Age/sex } & \multirow{2}{*}{ 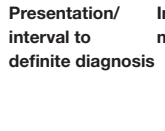 } & \multirow{2}{*}{$\begin{array}{l}\text { Initial } \\
\text { misdiagnosis }\end{array}$} & \multirow[t]{2}{*}{$\begin{array}{l}\text { DAVF } \\
\text { location }\end{array}$} & \multirow{2}{*}{$\begin{array}{l}\text { Concurrent with } \\
\text { venous sinus } \\
\text { stenosis/ } \\
\text { occlusion }\end{array}$} & \multicolumn{5}{|c|}{ Signal alteration on MRI } & \multirow{2}{*}{$\begin{array}{l}\text { Region of } \\
\text { congestion } \\
\text { al } \\
\text { id }\end{array}$} & \multirow{2}{*}{$\begin{array}{l}\text { Feeding } \\
\text { artery }\end{array}$} & \multirow[t]{2}{*}{ Draining vein } & \multirow[t]{2}{*}{$\begin{array}{l}\text { Cognard } \\
\text { classificatio }\end{array}$} & \multirow[t]{2}{*}{$\begin{array}{l}\text { Treatment } \\
\text { on }\end{array}$} & \multirow{2}{*}{$\begin{array}{l}\text { Degree of } \\
\text { DAVF } \\
\text { obliteration }\end{array}$} & \multirow[t]{2}{*}{$\begin{array}{l}\text { Follow-up } \\
\text { period }\end{array}$} & \multirow[t]{2}{*}{ Retreatment } & \multirow[t]{2}{*}{$\begin{array}{l}\text { t Outcome } \\
\text { (mRS) }\end{array}$} \\
\hline & & & & & & & $\mathrm{T} 1, \mathrm{~T} 1 \mathrm{C}_{+}$ & T2 & FLAIR & $\begin{array}{l}\text { DWI, } \\
\text { ADC }\end{array}$ & $\begin{array}{l}\text { Abnormal } \\
\text { vascular } \\
\text { flow-void }\end{array}$ & & & & & & & & & \\
\hline 1 & Probst et al. (5) & $40 / F$ & $\begin{array}{l}\text { Headache, nausea, } \\
\text { and } \\
\text { dnormalrientation/ } \\
\text { NANM }\end{array}$ & $\begin{array}{l}\text { Yes } \\
\text { (brain tumor) }\end{array}$ & TS & Yes & $\begin{array}{l}\text { NANM, } \\
\text { inhomogeneous } \\
\text { enhancement }\end{array}$ & $\begin{array}{l}\text { Hyper } \\
\text { is }\end{array}$ & NA/NM & $\begin{array}{l}\text { NA/NM, } \\
\text { NA/NM }\end{array}$ & Yes & $\begin{array}{l}\text { Pons, cerebellum, } \\
\text { and thalamus }\end{array}$ & $\begin{array}{l}\text { OA and } \\
\text { branches of } \\
\text { the ICA }\end{array}$ & $\begin{array}{l}\text { Straight } \\
\text { sinus } \rightarrow \text { vein of } \\
\text { Galen } \rightarrow \\
\text { pontomesencephalic } \\
\text { vein } \rightarrow \text { vein of } \\
\text { Rosenthal }\end{array}$ & $\begin{array}{l}\text { Type V } \\
\text { ic }\end{array}$ & $\begin{array}{l}\begin{array}{l}\text { Endovascular + } \\
\text { surgical }\end{array} \\
\end{array}$ & Completely & NANM & No & 0 \\
\hline 2 & Uchino et al. (6) & 68/F & $\begin{array}{l}\text { Gait disturbance, } \\
\text { dysarthria, and } \\
\text { urinary } \\
\text { incontinence/4 } \\
\text { years }\end{array}$ & No & CS & Yes & $\begin{array}{l}\text { Hypo, } \\
\text { enhanced }\end{array}$ & Hyper & NA/NM & $\begin{array}{l}\text { NAANM, } \\
\text { NA/NM }\end{array}$ & Yes & Pons & $\begin{array}{l}\text { Branches of } \\
\text { ECA and ICA }\end{array}$ & $\begin{array}{l}\text { Vein of Rosenthal, } \\
\text { inferior anastomotic } \\
\text { vein of Labbe, } \\
\text { pontine venous } \\
\text { congestion }\end{array}$ & Type IIB & $\begin{array}{l}\text { Subtotal TAE of In } \\
\text { ECA branches with } \\
\text { polyninyl alcohol } \\
\text { particles }\end{array}$ & Incompletely & 2 years & No & 5 \\
\hline 3 & & $74 / \mathrm{M}$ & $\begin{array}{l}\text { Chemosis, } \\
\text { proptosis, and gait } \\
\text { disturbance/ } \\
\text { NANM }\end{array}$ & No & CS & Yes & $\begin{array}{l}\text { Hypo, } \\
\text { enhanced }\end{array}$ & Hyper & NA/NM & $\begin{array}{l}\text { NAANM, } \\
\text { NA/NM }\end{array}$ & Yes & $\begin{array}{l}\text { Pons and } \\
\text { cerebellum }\end{array}$ & $\begin{array}{l}\text { Branches of } \\
\text { ECA and ICA }\end{array}$ & $\begin{array}{l}\text { Cortical veins of } \\
\text { the posterior fossa, } \\
\text { pontine venous } \\
\text { congestion }\end{array}$ & Type \|A+B & $\begin{array}{l}\text { Subtotal TAE of In } \\
\text { ECA branches with } \\
\text { polyvinyl alcohol } \\
\text { particles }\end{array}$ & Incompletely & 4 months & No & 2 \\
\hline 4 & Ernst et al. (7) & $71 / \mathrm{M}$ & $\begin{array}{l}\text { Paraparesis, } \\
\text { nausea, and } \\
\text { vomiting/ NA/NM }\end{array}$ & No & SPS & No & $\begin{array}{l}\text { NA/NM, } \\
\text { NA/NM }\end{array}$ & Hyper & NA/NM & $\begin{array}{l}\text { NA/NM, } \\
\text { NA/NM }\end{array}$ & Yes & $\begin{array}{l}\text { Hypoer medulla } \\
\text { oblongata } \\
\text { extending to the } \\
\text { upper cervical } \\
\text { cord }\end{array}$ & $\begin{array}{l}\text { MHT of } \\
\text { the ICA }\end{array}$ & PMV & Type V & Open surgery & Completely & 18 months & No & 1 \\
\hline 5 & & $58 / \mathrm{F}$ & $\begin{array}{l}\text { Tetrapraresis/many N } \\
\text { years }\end{array}$ & & $\mathrm{coJ}$ & No & $\begin{array}{l}\text { NA/NM, } \\
\text { NA/NM }\end{array}$ & Hyper & NA/NM & $\begin{array}{l}\text { NA/NM, } \\
\text { NA/NM }\end{array}$ & Yes & $\begin{array}{l}\text { Medulla } \\
\text { oblongata } \\
\text { extending to } \\
\text { the entire } \\
\text { cervical cord }\end{array}$ & $\begin{array}{l}\text { Ascending } \\
\text { cervical artery } \\
\text { of ECA, VA, } \\
\text { ophthalmic } \\
\text { artery }\end{array}$ & PMV & Type V & $\begin{array}{l}\text { TAE with PVA and In } \\
\text { silk thread }\end{array}$ & Incompletely & 4 years & No & 4 or 5 \\
\hline 6 & Chen et al. (2) & $47 / M$ & $\begin{array}{l}\text { Tetrapareis, } \\
\text { paresthesia, urinary } \\
\text { retention/1 year }\end{array}$ & No & Torcular & No & $\begin{array}{l}\text { NAINM, } \\
\text { NANMM }\end{array}$ & NAINM & NAINM & $\begin{array}{l}\text { NA/NM, } \\
\text { NA/NM }\end{array}$ & Yes & $\begin{array}{l}\text { Hypoer medulla } \\
\text { oblongata } \\
\text { extending to the } \\
\text { upper cervical } \\
\text { cord }\end{array}$ & $\begin{array}{l}\text { Meningeal } \\
\text { branch of the } \\
\text { VA }\end{array}$ & $\begin{array}{l}\text { Cerebellar } \\
\text { vein } \rightarrow \text { veins of the } \\
\text { hypoer } \\
\text { brainstem } \rightarrow \text { PMV }\end{array}$ & Type V & Open surgery & Completely & 2 months & No & 4 or 5 \\
\hline 7 & Ricolfi et al. (8) & $53 / \mathrm{M}$ & $\begin{array}{l}\text { Paraparesis, } \\
\text { paresthesia, urinary } \\
\text { retention/several } \\
\text { months }\end{array}$ & No & Tentorium & No & $\begin{array}{l}\text { Hypo, } \\
\text { non-enhanced }\end{array}$ & Hyper & NA/NM & $\begin{array}{l}\text { NAANM, } \\
\text { NA/NM }\end{array}$ & Yes & $\begin{array}{l}\text { Hypoer medulla } \\
\text { oblongata } \\
\text { extending to the } \\
\text { upper cervical } \\
\text { cord }\end{array}$ & $\begin{array}{l}\text { MHT of the } \\
\text { ICA, MMA }\end{array}$ & $\begin{array}{l}\text { Lateral } \\
\text { pontomesencephalic } \\
\text { veins } \rightarrow \text { cervical } \\
\text { and thoracic PMV }\end{array}$ & $\begin{array}{l}\text { Type V } \\
\text { ic }\end{array}$ & $\begin{array}{l}\text { TAE with NBCA via In } \\
\text { MMA and } \\
\text { occluding ICA }\end{array}$ & Incompletely & 2 years & $\begin{array}{l}\text { Yes/ } \\
\text { coagulated } \\
\text { the draining } \\
\text { veins }\end{array}$ & 1 or 2 \\
\hline 8 & & 40/F & $\begin{array}{l}\text { Tetrapareis, } \\
\text { sphincter } \\
\text { disturbance, bulbar } \\
\text { signs/ year }\end{array}$ & No & CS & No & $\begin{array}{l}\text { Hypo, } \\
\text { non-enhanced }\end{array}$ & Hyper & NA/NM & $\begin{array}{l}\text { NA/NM, } \\
\text { NA/NM }\end{array}$ & Yes & $\begin{array}{l}\text { Hypoer medulla } \\
\text { oblongata } \\
\text { extending to the } \\
\text { upper cervical } \\
\text { cord }\end{array}$ & $\begin{array}{l}\text { MHT of the } \\
\text { ICA, MMA, } \\
\text { sphenopalatine } \\
\text { artery and } \\
\text { AphA }\end{array}$ & $\begin{array}{l}\text { Superior } \\
\text { ophthalmic vein } \\
\text { eand SPS } \rightarrow \text { lateral } \\
\text { mesencephalic } \\
\text { veins } \rightarrow \text { PMV }\end{array}$ & Type V & $\begin{array}{l}\text { TAE with NBCA via } \mathrm{O} \\
\text { MMA and } \\
\text { sphenopalatine } \\
\text { arteries, with PVA } \\
\text { particles via APhA }\end{array}$ & Completely & 5 days & No & Dead \\
\hline 9 & & $75 / \mathrm{M}$ & $\begin{array}{l}\text { Tetraplegia, } \\
\text { sphincter } \\
\text { disturbance, bulbar } \\
\text { signs, } \\
\text { dysautonomia/a } \\
\text { few days }\end{array}$ & No & SPS & Yes & $\begin{array}{l}\text { Hypo, } \\
\text { non-enhanced }\end{array}$ & Hyper & NA/NM & $\begin{array}{l}\text { NAINM, I } \\
\text { NA/NM }\end{array}$ & No & $\begin{array}{l}\text { Hypoer pons and } 1 \\
\text { medulla oblongata } \\
\text { extending to the } \\
\text { upper cervical } \\
\text { cord }\end{array}$ & MMA & PMV & Type V & $\begin{array}{l}\text { TAE with NBCA via } 0 \\
\text { MMA }\end{array}$ & Completely & 5 years & No & 0 \\
\hline 10 & & $51 / / F$ & $\begin{array}{l}\text { Paraparesis, } \\
\text { sphincter } \\
\text { disturbance, bulbar } \\
\text { signs, } \\
\text { dysautonomia/3 } \\
\text { months }\end{array}$ & $\begin{array}{l}\text { Yes (initial } \\
\text { negative) }\end{array}$ & sS & Yes & $\begin{array}{l}\text { NA/NM, } \\
\text { NA/NM }\end{array}$ & Hyper & NA/NM & $\begin{array}{l}\text { NAANM, } \\
\text { NA/NM }\end{array}$ & Yes & $\begin{array}{l}\text { Medulla } \\
\text { oblongata } \\
\text { extending to } \\
\text { the upper cervical } \\
\text { cord }\end{array}$ & OA, MMA & $\begin{array}{l}\text { Lateral medullary } \\
\text { vein } \rightarrow \text { PMV }\end{array}$ & Type V & $\begin{array}{l}\text { TAE with NBCA via } \mathrm{C} \\
\text { MMA and OA }\end{array}$ & Completely & 1 year & No & 0 \\
\hline 11 & Bousson et al. (9) & $36 / \mathrm{M}$ & $\begin{array}{l}\text { Tetrapraresis, } \\
\text { paresthesia/4 } \\
\text { months }\end{array}$ & No & Tentorium & No & $\begin{array}{l}\text { NA/NM, } \\
\text { intensely } \\
\text { enhanced }\end{array}$ & Hyper & NA/NM & $\begin{array}{l}\text { NA/NM, } \\
\text { NA/NM }\end{array}$ & Yes & $\begin{array}{l}\text { Medulla } \\
\text { oblongata } \\
\text { extending to } \\
\text { the entire cervical } \\
\text { cord }\end{array}$ & $\mathrm{OA}$ & $\begin{array}{l}\text { Vein around } \\
\text { brainstem } \rightarrow \text { PMV }\end{array}$ & Type V & $\begin{array}{l}\text { TAE to occlude the In } \\
\text { OA }\end{array}$ & Incompletely & 2 weeks & No & NANM \\
\hline
\end{tabular}


TABLE 1 | Continued

\begin{tabular}{|c|c|c|c|c|c|c|c|c|c|c|c|c|c|c|c|c|c|c|c|}
\hline \multirow{3}{*}{$\begin{array}{l}\text { No.Author/year } \\
12 \text { Hurst et al. (10) }\end{array}$} & \multirow{3}{*}{$\begin{array}{c}\text { Age/sex } \\
\\
54 / \mathrm{M}\end{array}$} & \multirow{3}{*}{$\begin{array}{l}\begin{array}{l}\text { Presentation/ } \\
\text { interval to } \\
\text { definite diagnosis }\end{array} \\
\begin{array}{l}\text { Tetrapraresis/ } \\
\text { NANM }\end{array}\end{array}$} & \multirow{3}{*}{$\begin{array}{l}\text { Initial } \\
\text { misdiagnosis } \\
\text { No }\end{array}$} & \multirow{3}{*}{$\begin{array}{l}\text { DAVF } \\
\text { location } \\
\text { COJ }\end{array}$} & \multirow{3}{*}{$\begin{array}{l}\text { Concurrent with } \\
\text { venous sinus } \\
\text { stenosis/ } \\
\text { occlusion }\end{array}$} & \multicolumn{5}{|c|}{ Signal alteration on MRI } & \multirow{2}{*}{$\begin{array}{l}\text { Region of } \\
\text { congestion } \\
\text { I }\end{array}$} & \multirow{2}{*}{$\begin{array}{l}\text { Feeding } \\
\text { artery }\end{array}$} & \multirow{2}{*}{ Draining vein } & \multirow{2}{*}{$\begin{array}{l}\text { Cognard } \\
\text { classificatio }\end{array}$} & \multirow{2}{*}{$\begin{array}{l}\text { Treatment } \\
\text { on }\end{array}$} & \multirow{2}{*}{$\begin{array}{l}\text { Degree of } \\
\text { DAVF } \\
\text { obliteration }\end{array}$} & \multirow{2}{*}{$\begin{array}{l}\text { Follow-up } \\
\text { period }\end{array}$} & \multirow{2}{*}{ Retreatment } & \multirow{2}{*}{$\begin{array}{l}\text { t Outcome } \\
\text { (mRS) }\end{array}$} \\
\hline & & & & & & $\mathrm{T} 1, \mathrm{~T} 1 \mathrm{C}+$ & T2 & FLAIR & $\begin{array}{l}\text { DWI, } \\
\text { ADC }\end{array}$ & $\begin{array}{l}\text { Abnormal } \\
\text { vascular } \\
\text { flow-void }\end{array}$ & & & & & & & & & \\
\hline & & & & & & $\begin{array}{l}\text { NA/NM, } \\
\text { enhanced }\end{array}$ & Hyper & NAANM & $\begin{array}{l}\text { NANNM } \\
\text { NA/NM }\end{array}$ & Yes & $\begin{array}{l}\text { Hypoer medulla } \\
\text { oblongata } \\
\text { extending to the } \\
\text { upper cervical } \\
\text { cord }\end{array}$ & $\begin{array}{l}\text { Dural branch } \\
\text { of VA }\end{array}$ & PMV & Type V & $\begin{array}{l}\text { TAE with PVA via } \\
\text { the dural branch of } \\
\text { VA }\end{array}$ & Completely & 3 months & No & 3 \\
\hline 13 & $50 / \mathrm{M}$ & $\begin{array}{l}\text { Tetrapraresis, pain, } 1 \\
\text { hypoer CN deficits/ } \\
\text { NANMM }\end{array}$ & & COJ & № & Hypo/ NA/NM & Hyper & NANM & $\begin{array}{l}\text { NA/NM/ } \\
\text { NA/NM }\end{array}$ & Yes & $\begin{array}{l}\text { Hypoer medulla } \\
\text { oblongata } \\
\text { extending to the } \\
\text { upper cervical } \\
\text { cord }\end{array}$ & AphA & PMV & Type V & $\begin{array}{l}\text { TAE with polyvinyl } \\
\text { alcohol via APhA }\end{array}$ & Completely & 12 months & No & 4 \\
\hline $\begin{array}{l}14 \text { Takahashi et al. } \\
\text { (11) }\end{array}$ & $49 / \mathrm{M}$ & $\begin{array}{l}\text { Diplopia, vertigo/3 } \\
\text { weeks }\end{array}$ & No & cs & Yes & $\begin{array}{l}\text { Hypo, } \\
\text { enhanced }\end{array}$ & Hyper & NANM & $\begin{array}{l}\text { NAANM, } \\
\text { NAANM }\end{array}$ & Yes & $\begin{array}{l}\text { Pons and } \\
\text { cerebellar } \\
\text { hemisphere }\end{array}$ & $\begin{array}{l}\text { MHT of } \\
\text { the ICA }\end{array}$ & $\begin{array}{l}\text { SPS } \rightarrow \text { ophthalmic T } \\
\text { vein, petrosal } \\
\text { vein } \rightarrow \text { cortical } \\
\text { venous reflux }\end{array}$ & Type \|A+B & TVE with coils & Completely & 3 months & No & 0 or 1 \\
\hline 15 & $62 / \mathrm{F}$ & $\begin{array}{l}\text { Loss of visual } \\
\text { acuity, chemosis, } \\
\text { exophthalmos/ } \\
\text { NA/NM }\end{array}$ & No & cs & № & $\begin{array}{l}\text { Hypo, } \\
\text { markedly } \\
\text { enhanced }\end{array}$ & Hyper & NANM & $\begin{array}{l}\text { NANM, } \\
\text { NANMM }\end{array}$ & Yes & $\begin{array}{l}\text { Pons and } \\
\text { medulla } \\
\text { oblongata }\end{array}$ & $\begin{array}{l}\text { Branches of } \\
\text { bilateral ECA } \\
\text { and ICA }\end{array}$ & $\begin{array}{l}\text { CS } \rightarrow \text { superior } \\
\text { ophthalmic vein }\end{array}$ & Type \|A+B & TVE with coils & Completely & 1 month & No & 2 \\
\hline $\begin{array}{l}16 \text { Shintani et al. } \\
\text { (12) }\end{array}$ & $65 / \mathrm{F}$ & $\begin{array}{l}\text { Chemosis, CN (III, I } \\
\text { IV, VI) palsy, } \\
\text { vertigo/8 months }\end{array}$ & No & cs & № & $\begin{array}{l}\text { Hypo, } \\
\text { markedly } \\
\text { enhanced }\end{array}$ & Hyper & NANM & $\begin{array}{l}\text { NA/NM, } \\
\text { NAANM }\end{array}$ & & Pons & $\begin{array}{l}\text { Branches of } \\
\text { ICA }\end{array}$ & IPS & NAINM & NAINM & NANM & NANM & NANM & Dead \\
\hline $\begin{array}{l}17 \text { Wiesmann et al. } \\
\text { (13) }\end{array}$ & 46/M & $\begin{array}{l}\text { Paraparesis, } \\
\text { dysarthria, urinary } \\
\text { incontinence/4 } \\
\text { days }\end{array}$ & No & COJ & Yes & $\begin{array}{l}\text { NAANM, } \\
\text { NANM }\end{array}$ & Hyper & NANM & $\begin{array}{l}\text { NA/NM, } \\
\text { NA/NM }\end{array}$ & & $\begin{array}{l}\text { Pontomedullary } \\
\text { region }\end{array}$ & NMB of AphA & $\begin{array}{l}\text { Anterior median } \\
\text { pontine and } \\
\text { anterior medullary } \\
\text { veins anterior and } \\
\text { posterior spinal } \\
\text { veins }\end{array}$ & Type V & $\begin{array}{l}\text { TAE with NBCA via } C \\
\text { AphA }\end{array}$ & Completely & 12 months & No & 1 \\
\hline $\begin{array}{l}18 \text { Kalamangalam } \\
\text { et al. (14) }\end{array}$ & $68 / \mathrm{M}$ & $\begin{array}{l}\text { Paraparesis, } \\
\text { urinary } \\
\text { incontinence/4 } \\
\text { months }\end{array}$ & Yes (stroke) & $\cos$ (Clivus) $N$ & & $\begin{array}{l}\text { Normal, } \\
\text { non-enhanced }\end{array}$ & Hyper & NANM & $\begin{array}{l}\text { NA/NM, } \\
\text { NAANM }\end{array}$ & & $\begin{array}{l}\text { Hypoer medulla } \\
\text { oblongata } \\
\text { extending to the } \\
\text { entire cervical } \\
\text { cord }\end{array}$ & $\begin{array}{l}\text { MHT of } \\
\text { the ICA }\end{array}$ & $\begin{array}{l}\text { Veins around } \\
\text { brainstem } \\
\text { cervical spinal cord }\end{array}$ & Type V & $\begin{array}{l}\text { Surgical clipping } \\
\text { draining vein }\end{array}$ & Completely & 4 months & No & 3 \\
\hline $\begin{array}{l}19 \text { Weigele et al. } \\
\text { (15) }\end{array}$ & $53 / \mathrm{M}$ & $\begin{array}{l}\text { Cranial } \\
\text { neuropathies, } \\
\text { hemidysesthesia, } \\
\text { and personality } \\
\text { changes/several } \\
\text { months }\end{array}$ & $\begin{array}{l}\text { Yes (brainstem } \\
\text { glioma) }\end{array}$ & Galen vein $N$ & № & $\begin{array}{l}\text { Normal, } \\
\text { non-enhanced }\end{array}$ & Hyper & Hyper & $\begin{array}{l}\text { NA/NM, } \\
\text { NA/NM }\end{array}$ & & $\begin{array}{l}\text { Pons, midbrain, } \\
\text { and thalamus }\end{array}$ & $\begin{array}{l}\text { MMA, NMB } \\
\text { of AphA, } \\
\text { marginal artery, } \\
\text { vermin branch s } \\
\text { of SCA }\end{array}$ & $\begin{array}{l}\text { Pontomedullary } \\
\text { and anterior cortical } \\
\text { veins superior } \\
\text { sagittal sinus }\end{array}$ & Type IV & $\begin{array}{l}\text { TAE with NBCA via } \\
\text { MMA and AphA }\end{array}$ & Completely & 6 months & No & 0 \\
\hline $\begin{array}{l}20 \text { Asakawa et al. } \\
\text { (16) }\end{array}$ & $64 / \mathrm{M}$ & $\begin{array}{l}\text { Tetrapraresis, } \\
\text { urinary } \\
\text { incontinence, } \\
\text { respiratory } \\
\text { insufficiency/2 } \\
\text { weeks }\end{array}$ & No & $\begin{array}{l}\subset C J \\
\text { (foramen } \\
\text { magnum) }\end{array}$ & № & $\begin{array}{l}\text { Hypo, } \\
\text { enhanced }\end{array}$ & Hyper & NANM & $\begin{array}{l}\text { NA/NM, } \\
\text { NA/NM }\end{array}$ & & $\begin{array}{l}\text { Hypoer medulla } \\
\text { oblongata } \\
\text { extending to the } \\
\text { upper thoracic } \\
\text { cord }\end{array}$ & AphA & Spinal veins & Type V & $\begin{array}{l}\text { Combined TAE and } \mathrm{C} \\
\text { surgical interuption }\end{array}$ & $\begin{array}{l}\text { d Completely } \\
\text { n }\end{array}$ & 3 months & No & 4 \\
\hline 21 Lanz et al. (17) & 68/F & $\begin{array}{l}\text { Diplopia, } \\
\text { dysarthria, } \\
\text { syncope, transient } \\
\text { Paraparesis, } \\
\text { respiratory } \\
\text { insufficiency/1/ year }\end{array}$ & No & ss & Yes & $\begin{array}{l}\text { Normal, } \\
\text { non-enhanced }\end{array}$ & Hyper & NANM & $\begin{array}{l}\text { NAANM, } \\
\text { NANMM }\end{array}$ & & $\begin{array}{l}\text { Medulla oblongata } \\
\text { extending to the } \\
\text { upper cervical } \\
\text { cord }\end{array}$ & & $\begin{array}{l}S S \rightarrow \text { vein around } \\
\text { brainstem } \rightarrow \text { PMV }\end{array}$ & Type V & $\begin{array}{l}\text { TAE with NBCA via } 0 \\
\text { MMA }\end{array}$ & Completely & NM & No & 0 \\
\hline 22 Kai et al. (18) & $56 / \mathrm{F}$ & $\begin{array}{l}\text { Proptosis, double } \\
\text { vision, visual } \\
\text { disturbance, } \\
\text { hemiparesis/2 } \\
\text { weeks }\end{array}$ & No & CS & № & $\begin{array}{l}\text { NA/NM, } \\
\text { moderately } \\
\text { enhanced }\end{array}$ & Hyper & NAINM & $\begin{array}{l}\text { NAANM, } \\
\text { NA/NM }\end{array}$ & & Brainstem & $\begin{array}{l}\text { Branches of } \\
\text { the ECA }\end{array}$ & 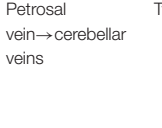 & Type IIA+B & $\begin{array}{l}\text { TVE via petrosal In } \\
\text { vein cannulation } \\
\text { with coils }\end{array}$ & Incompletely & 1 month & No & 3 \\
\hline 23 & 70/F & $\begin{array}{l}\text { Double vision, } \\
\text { chemosis, } \\
\text { exophthalmos, } \\
\text { ataxia/2 months }\end{array}$ & No & CS & Yes & $\begin{array}{l}\text { Normal, } \\
\text { non-enhanced }\end{array}$ & Hyper & NANM & $\begin{array}{l}\text { NAANM, } \\
\text { NAANM }\end{array}$ & & Midbrain & $\begin{array}{l}\text { Dural } \\
\text { branches of thes } \\
\text { bilateral ICAs } \\
\text { and ECAs }\end{array}$ & $\begin{array}{l}\text { Sphenoparietal } \\
\text { esinus } \rightarrow \text { deep } \\
\text { sylvan vein } \rightarrow \\
\text { pontomesencephalic } \\
\text { veins }\end{array}$ & $\begin{array}{l}\text { Type IIA+B } \\
\text { ic }\end{array}$ & $\begin{array}{l}\text { Packing of CS with C } \\
\text { sponges via open } \\
\text { surgery }\end{array}$ & & 1 months & No & 0 \\
\hline
\end{tabular}


TABLE 1 | Continued

\begin{tabular}{|c|c|c|c|c|c|c|c|c|c|c|c|c|c|c|c|c|c|c|c|}
\hline \multirow[t]{2}{*}{ No.Author/year } & \multirow[t]{2}{*}{ Age/sex } & \multirow{2}{*}{$\begin{array}{l}\text { Presentation/ } \\
\text { interval to } \\
\text { definite diagnosis }\end{array}$} & \multirow{2}{*}{$\begin{array}{l}\text { Initial } \\
\text { misdiagnosis } \\
s\end{array}$} & \multirow{2}{*}{$\begin{array}{l}\text { DAVF } \\
\text { location }\end{array}$} & \multirow{2}{*}{$\begin{array}{l}\text { Concurrent with } \\
\text { venous sinus } \\
\text { stenosis/ } \\
\text { occlusion }\end{array}$} & \multicolumn{5}{|c|}{ Signal alteration on MRI } & \multirow{2}{*}{$\begin{array}{l}\text { Region of } \\
\text { congestion } \\
\text { l }\end{array}$} & \multirow[t]{2}{*}{$\begin{array}{l}\text { Feeding } \\
\text { artery }\end{array}$} & \multirow[t]{2}{*}{ Draining vein } & \multirow[t]{2}{*}{$\begin{array}{l}\text { Cognard } \\
\text { classificatior }\end{array}$} & \multirow[t]{2}{*}{$\begin{array}{l}\text { Treatment } \\
\text { on }\end{array}$} & \multirow{2}{*}{$\begin{array}{l}\text { Degree of } \\
\text { DAVF } \\
\text { obliteration }\end{array}$} & \multirow[t]{2}{*}{$\begin{array}{l}\text { Follow-up } \\
\text { period }\end{array}$} & \multirow[t]{2}{*}{ Retreatment } & \multirow[t]{2}{*}{$\begin{array}{l}\text { Outcome } \\
\text { (mRS) }\end{array}$} \\
\hline & & & & & & $\mathrm{T} 1, \mathrm{~T} 1 \mathrm{C}+$ & T2 & FLAIR & $\begin{array}{l}\text { DWI, } \\
\text { ADC }\end{array}$ & $\begin{array}{l}\text { Abnormal } \\
\text { vascular } \\
\text { flow-void }\end{array}$ & & & & & & & & & \\
\hline 24 Li et al. (19) & $73 / \mathrm{M}$ & $\begin{array}{l}\text { Tetrapraresis, } \\
\text { unconsciousness } \\
\text { and dyspnea/1 yeari }\end{array}$ & $\begin{array}{l}\text { Yes (acute } \\
\text { cerebral } \\
\text { arinfarction) }\end{array}$ & TS & Yes & $\begin{array}{l}\text { NA/NM, } \\
\text { NA/NM }\end{array}$ & Hyper & Hyper & $\begin{array}{l}\text { NANM, } \\
\text { NANMM }\end{array}$ & Yes & $\begin{array}{l}\text { Temporal lobe } \\
\text { and medulla } \\
\text { oblongata } \\
\text { extending to the } \\
\text { upper thoracic } \\
\text { cord }\end{array}$ & $\begin{array}{l}\text { MMA, OA, } \\
\text { AphA }\end{array}$ & $\begin{array}{l}\text { Cortical vein, } \\
\text { stenotic } \\
\text { TS } \rightarrow \text { anterior and } \\
\text { posterior spinal vein }\end{array}$ & $\begin{array}{l}\text { Type V } \\
\text { n }\end{array}$ & $\begin{array}{l}\text { TVE with coiling the } \\
\text { TS }\end{array}$ & Completely & 5 days & No & NANM \\
\hline 25 Pannu et al. (20) & $42 / \mathrm{M}$ & $\begin{array}{l}\text { Tetrapraresis, } \\
\text { bowel and urinary } \\
\text { incontinence// year }\end{array}$ & No & Tentorium & No & $\begin{array}{l}\text { NA/NM, } \\
\text { NA/NM }\end{array}$ & Hyper & Hyper & $\begin{array}{l}\text { NA/NM, } \\
\text { NA/NM }\end{array}$ & Yes & $\begin{array}{l}\text { Medulla } \\
\text { oblongata } \\
\text { extending to the } \\
\text { upper cervical } \\
\text { cord }\end{array}$ & $\begin{array}{l}\text { MHT of } \\
\text { the ICA }\end{array}$ & $\begin{array}{l}\text { Superior petrosal } \\
\text { vein } \rightarrow \text { ateral } \\
\text { medullary vein } \rightarrow \\
\text { the anterior and } \\
\text { posterior spinal } \\
\text { veins }\end{array}$ & Type V & $\begin{array}{l}\text { Coagulating DAVF } \\
\text { and draining vein }\end{array}$ & Completely & 12 months & No & 3 \\
\hline 26 Crum et al. (21) & $35 / \mathrm{M}$ & $\begin{array}{l}\text { Paraparesis, } \\
\text { ataxia, } \\
\text { diplopia/several } \\
\text { weeks }\end{array}$ & $\begin{array}{l}\text { Yes (uncertain } \\
\text { brainstem lesior }\end{array}$ & $\begin{array}{l}\text { CCJ (jugular } \\
\text { 1) foramen) }\end{array}$ & r No & $\begin{array}{l}\text { Normal, patchy } \\
\text { enhancement }\end{array}$ & y Hyper & Hyper & $\begin{array}{l}\text { NANM, } \\
\text { NANMM }\end{array}$ & Yes & $\begin{array}{l}\text { Medulla } \\
\text { oblongata } \\
\text { extending to the } \\
\text { upper cervical } \\
\text { cord }\end{array}$ & $\begin{array}{l}\text { Branches of } \\
\text { the VA and } \\
\text { PICA }\end{array}$ & $\begin{array}{l}\text { Spinal medullary } \\
\text { veins }\end{array}$ & Type V & $\begin{array}{l}\text { Coagulated and } \\
\text { divided the DAVF } \\
\text { and draining vein }\end{array}$ & Completely & 3 months & No & 1 \\
\hline 27 Oishi et al. (22) & $68 / F$ & $\begin{array}{l}\text { Disturbance of } \\
\text { brainstem } \\
\text { function/NA/NM }\end{array}$ & NANM & TS & NAINM & $\begin{array}{l}\text { NANM, } \\
\text { NANMM }\end{array}$ & Hyper & Hyper & $\begin{array}{l}\text { NANM, } \\
\text { NA/NM }\end{array}$ & Yes & $\begin{array}{l}\text { Medulla } \\
\text { oblongata }\end{array}$ & NANM & SPS $\rightarrow$ spinal PMV & Type V & TVE with coils & Completely & NAINM & № & NA/NM \\
\hline 28 Satoh et al. (23) & $38 / F$ & $\begin{array}{l}\text { Tetrapraresis, } \\
\text { nystagmus, } \\
\text { Horner } \\
\text { syndrome/NA/NM }\end{array}$ & No & TS-SS & Yes & Hypo, NAINM & Hyper & Hyper & $\begin{array}{l}\text { NANM, } \\
\text { NANMM }\end{array}$ & No & $\begin{array}{l}\text { Medulla } \\
\text { oblongata }\end{array}$ & $\begin{array}{l}\text { MMA, OA, } \\
\text { AphA, MHT of } \\
\text { the ICA, PMA } \\
\text { of the VA }\end{array}$ & SS $\rightarrow$ spinal PMV & Type V & $\begin{array}{l}\text { TVE with coiling the } \\
\text { SS }\end{array}$ & Completely & 1 month & No & 3 \\
\hline 29 Tanoue et al. (3) & $70 / \mathrm{M}$ & $\begin{array}{l}\text { Tetrapraresis, } \\
\text { sensory } \\
\text { disturbance/ } \\
2 \text { years }\end{array}$ & No & $\begin{array}{l}\mathrm{CCJ} \\
\text { (foramen } \\
\text { magnum) }\end{array}$ & No & $\begin{array}{l}\text { Normal, } \\
\text { non-enhanced }\end{array}$ & Hyper & NA/NM & $\begin{array}{l}\text { NANM, } \\
\text { NANMM }\end{array}$ & Yes & $\begin{array}{l}\text { Medulla } \\
\text { oblongata } \\
\text { extending to the } \\
\text { entire cervical } \\
\text { cord }\end{array}$ & $\begin{array}{l}\text { Jugular } \\
\text { branch of OA, } \\
\mathrm{NMH} \text { of AphA }\end{array}$ & $\begin{array}{l}\text { Anterior condylar } \\
\text { vein } \rightarrow \text { inferior } \\
\text { petrosall } \\
\text { sinus } \rightarrow \text { pontomesen } \\
\text { vein } \rightarrow \text { anterior } \\
\text { spinal vein }\end{array}$ & Type V & $\begin{array}{l}\text { TAE with NBCA via } \\
\text { AphA and OA }\end{array}$ & Incompletely & 14 months & No & 4 or 5 \\
\hline 30 Akkoc et al. (24) & $45 / \mathrm{M}$ & $\begin{array}{l}\text { Paraparesis, } \\
\text { urinary retention/ } \\
2 \text { months }\end{array}$ & $\begin{array}{l}\text { Yes (brainstem } \\
\text { ischemia or } \\
\text { myelitis) }\end{array}$ & cos & No & $\begin{array}{l}\text { NA/NM, } \\
\text { NA/NM }\end{array}$ & Hyper & NA/NM & $\begin{array}{l}\text { NANM, } \\
\text { NANMM }\end{array}$ & Yes & $\begin{array}{l}\text { Medulla } \\
\text { oblongata } \\
\text { extending to the } \\
\text { entire cervical } \\
\text { cord }\end{array}$ & $\begin{array}{l}\text { OA, NMH of } \\
\text { AphA }\end{array}$ & PMV & Type V & $\begin{array}{l}\text { TAE with NBCA via } \\
\text { OA }\end{array}$ & Completely & 3 months & $\begin{array}{l}\text { Yes/repeated } \\
\text { TAE with } \\
\text { NBCA via } \\
\text { AphA }\end{array}$ & 4 or 5 \\
\hline 31 Iwasaki et al. (25) & $71 / \mathrm{F}$ & $\begin{array}{l}\text { Decreased } \\
\text { abduction of the } \\
\text { right eye/ } \\
5 \text { months }\end{array}$ & $\begin{array}{l}\text { Yes (brain } \\
\text { neoplasm) }\end{array}$ & CS & No & $\begin{array}{l}\text { Normal, } \\
\text { patchy- } \\
\text { enhancement }\end{array}$ & Hyper & NA/NM & $\begin{array}{l}\text { NAANM, } \\
\text { NAANM }\end{array}$ & No & Upper pons & $\begin{array}{l}\text { MMA, } \\
\text { meningeal } \\
\text { branch of the } \\
\text { ICA }\end{array}$ & $\begin{array}{l}\text { SPS } \rightarrow \text { straight } \\
\text { sinus } \rightarrow \text { cerebellar } \\
\text { cortical veins } \rightarrow \\
\text { anterior } \\
\text { pontomesencephalic } \\
\text { vein } \rightarrow \text { PMV }\end{array}$ & $\begin{array}{l}\text { Type V } \\
\text { lic }\end{array}$ & $\begin{array}{l}\text { Stereotactic } \\
\text { radiosurgery }\end{array}$ & Completely & 3 years & No & 0 \\
\hline $\begin{array}{l}32 \text { Lagares et al. } \\
\text { (26) }\end{array}$ & $65 / \mathrm{M}$ & $\begin{array}{l}\text { Tetrapraresis, } \\
\text { respiratory } \\
\text { insufficiency/3 } \\
\text { months }\end{array}$ & $\begin{array}{l}\text { Yes (cerebellar } \\
\text { infarction) }\end{array}$ & Torcular & No & $\begin{array}{l}\text { NANM, } \\
\text { NANMM }\end{array}$ & Hyper & Hyper & $\begin{array}{l}\text { Hypo, } \\
\text { hyper }\end{array}$ & Yes & $\begin{array}{l}\text { Medulla } \\
\text { oblongata }\end{array}$ & $\begin{array}{l}\text { OA, PMA of the } \\
\text { VA }\end{array}$ & $\begin{array}{l}\text { e Cerebellar } \\
\text { vein } \rightarrow \text { petrosal vein } \\
\text { and PMV }\end{array}$ & Type V & Open surgery & Completely & 6 months & No & 1 \\
\hline $\begin{array}{l}33 \text { van Rooij et al. } \\
(27)\end{array}$ & $58 / \mathrm{M}$ & $\begin{array}{l}\text { Tetrapraresis, } \\
\text { bladder retention/3 } \\
\text { months }\end{array}$ & Yes (NA/NM) & Tentorium & No & $\begin{array}{l}\text { NA/NM, } \\
\text { NA/NM }\end{array}$ & Hyper & NA/NM & $\begin{array}{l}\text { NANM, } \\
\text { NANMM }\end{array}$ & Yes & $\begin{array}{l}\text { Medulla } \\
\text { oblongata } \\
\text { extending to the } \\
\text { entire cervical } \\
\text { cord }\end{array}$ & $\begin{array}{l}\text { MHT of the } \\
\text { ICA, MMA, } \\
\text { AphA }\end{array}$ & $\begin{array}{l}\text { Petrosal } \\
\text { vein } \rightarrow \text { PMV }\end{array}$ & Type V & $\begin{array}{l}\text { TAE with NBCA via } \\
\text { MMA }\end{array}$ & Completely & 1 years & No & 0 \\
\hline 34 & $72 / \mathrm{F}$ & $\begin{array}{l}\text { Tetrapraresis, } \\
\text { paresthesias, } \\
\text { bladder retention/2 } \\
\text { years }\end{array}$ & Yes (NAINM) & $\begin{array}{l}\text { CCJ } \\
\text { (foramen } \\
\text { magnum) }\end{array}$ & No & $\begin{array}{l}\text { NA/NM, } \\
\text { NA/NM }\end{array}$ & Hyper & NA/NM & $\begin{array}{l}\text { NA/NM, } \\
\text { NA/NMM }\end{array}$ & Yes & $\begin{array}{l}\text { Medulla } \\
\text { oblongata } \\
\text { extending to the } \\
\text { middle thoracic } \\
\text { cord }\end{array}$ & $\mathrm{OA}$ & PMV & Type V & $\begin{array}{l}\text { TAE with NBCA via } \\
\text { OA }\end{array}$ & Completely & 2 years & No & 4 or 5 \\
\hline $\begin{array}{l}35 \text { Sakamoto et al. } \\
(28)\end{array}$ & 65/F & $\begin{array}{l}\text { Progressive mental } \\
\text { and gait } \\
\text { disturbance/1 } \\
\text { month }\end{array}$ & & TS-SS & Yes & Hypo, NA/NM & Hyper & NA/NM & $\begin{array}{l}\text { Hetero- } \\
\text { geneous, } \\
\text { hyper }\end{array}$ & & $\begin{array}{l}\text { Brainstem and } \\
\text { cerebellum }\end{array}$ & $\begin{array}{l}\text { OA, NMH of } \\
\text { AphA, posterior } \\
\text { branch of } \\
\text { MMA, anterior } \\
\text { and posterior } \\
\text { auricular } \\
\text { arteries }\end{array}$ & NA/NM & TypellB & TVE with coils & Completely & NAINM & No & 0 \\
\hline
\end{tabular}


TABLE 1 | Continued

\begin{tabular}{|c|c|c|c|c|c|c|c|c|c|c|c|c|c|c|c|c|c|c|c|}
\hline \multirow{3}{*}{$\begin{array}{l}\text { No.Author/year } \\
\begin{array}{l}36 \text { Tsutsumi et al. } \\
\text { (29) }\end{array}\end{array}$} & \multirow{3}{*}{$\begin{array}{c}\text { Age/sex } \\
62 / F \\
\end{array}$} & \multirow{3}{*}{$\begin{array}{l}\text { Presentation/ } \\
\text { interval to } \\
\text { definite diagnosis }\end{array}$} & \multirow{3}{*}{$\begin{array}{l}\text { Initial } \\
\text { misdiagnosis } \\
\text { Yes } \\
\text { (intramedullary } \\
\text { (glioma) }\end{array}$} & \multirow{3}{*}{$\begin{array}{l}\begin{array}{l}\text { DAVF } \\
\text { location }\end{array} \\
\begin{array}{l}\text { CCJ } \\
\text { (foramen } \\
\text { magnum) }\end{array}\end{array}$} & \multirow{3}{*}{$\begin{array}{l}\text { Concurrent with } \\
\text { venous sinus } \\
\text { stenosis/ } \\
\text { occlusion }\end{array}$} & \multicolumn{5}{|c|}{ Signal alteration on MRI } & \multirow{2}{*}{$\begin{array}{l}\text { Region of } \\
\text { congestion } \\
\text { a }\end{array}$} & \multirow{2}{*}{$\begin{array}{l}\text { Feeding } \\
\text { artery }\end{array}$} & \multirow{2}{*}{ Draining vein } & \multirow{2}{*}{$\begin{array}{l}\text { Cognard } \\
\text { classificatio }\end{array}$} & \multirow{2}{*}{$\begin{array}{l}\text { Treatment } \\
\text { ion }\end{array}$} & \multirow{2}{*}{$\begin{array}{l}\text { Degree of } \\
\text { DAVF } \\
\text { obliteration }\end{array}$} & \multirow{2}{*}{$\begin{array}{l}\text { Follow-up } \\
\text { period }\end{array}$} & \multirow[t]{2}{*}{ Retreatment } & \multirow{2}{*}{$\begin{array}{l}\text { toutcome } \\
\text { (mRS) }\end{array}$} \\
\hline & & & & & & $\mathrm{T} 1, \mathrm{~T} 1 \mathrm{C}+$ & T2 & FLAIR & $\begin{array}{l}\mathrm{DWI}, \\
\mathrm{ADC}\end{array}$ & $\begin{array}{l}\text { Abnorma } \\
\text { vascular } \\
\text { flow-void }\end{array}$ & & & & & & & & & \\
\hline & & & & & & $\begin{array}{l}\text { Hypo, rim-like } \\
\text { enhancement }\end{array}$ & Hyper & NAINM & $\begin{array}{l}\text { NAINM, } \\
\text { NA/NM }\end{array}$ & No & $\begin{array}{l}\text { Medulla } \\
\text { oblongata } \\
\text { extending to the } \\
\text { upper thoracic } \\
\text { cord }\end{array}$ & $\begin{array}{l}\mathrm{NMH} \text { of AphA, } \\
\text { meningeal } \\
\text { branch of OA }\end{array}$ & $\begin{array}{l}\text { Retrograde } \\
\text { drainage to the } \\
\text { inferior petrosal } \\
\text { sinus } \rightarrow \text { cavernous } \\
\text { sinuses }\end{array}$ & Type IIA & TVE with coils & Completely & Immediately I & No & NAINM \\
\hline $\begin{array}{l}37 \text { Sugiura et al. } \\
(30)\end{array}$ & $69 / \mathrm{F}$ & $\begin{array}{l}\text { Vomiting, ataxia } \\
\text { and weakness/2 } \\
\text { months }\end{array}$ & No & ss & Yes & $\begin{array}{l}\text { NANM, } \\
\text { patchy- } \\
\text { enhancement }\end{array}$ & Hyper & NA/NM & $\begin{array}{l}\text { Normal, } \\
\text { hyper }\end{array}$ & Yes & $\begin{array}{l}\text { Medulla } \\
\text { oblongata and } \\
\text { hypoer pons }\end{array}$ & $O A$ & $\begin{array}{l}\text { Veins around } \\
\text { brainstem } \rightarrow \text { spinal } \\
\text { PMV }\end{array}$ & Type V & $\begin{array}{l}\text { TVE with coiling } \\
\text { the SS }\end{array}$ & Completely & 3 weeks & No & 4 or 5 \\
\hline 38 Wang et al. (31) & $68 / \mathrm{M}$ & $\begin{array}{l}\text { Focal motor deficit/ } \\
\text { NAINM }\end{array}$ & NAINM & $\begin{array}{l}\text { CCJ } \\
\text { (foramen } \\
\text { magnum) }\end{array}$ & No & $\begin{array}{l}\text { NANM, } \\
\text { NANMM }\end{array}$ & Hyper & NA/NM & $\begin{array}{l}\text { NAINM, } \\
\text { NAINM, }\end{array}$ & Yes & $\begin{array}{l}\text { Medulla } \\
\text { oblongata }\end{array}$ & NMB of AphA & $\begin{array}{l}\text { PMV and } \\
\text { anteromedullary } \\
\text { cenvical veins }\end{array}$ & Type V & $\begin{array}{l}\text { TAE with NBCA via } \\
\text { AphA }\end{array}$ & a Completely & 2 years & No & 4 or 5 \\
\hline 39 Khan et al. (32) & $20 / F$ & $\begin{array}{l}\text { Tetrapraresis, } \\
\text { urinary retention } \\
\text { and respiratory } \\
\text { distress/1 month }\end{array}$ & $\begin{array}{l}\text { Yes } \\
\text { (demyelinating } \\
\text { disease) }\end{array}$ & Tentorium & No & $\begin{array}{l}\text { NA/NM, } \\
\text { non-enhanced }\end{array}$ & Hyper & Hyper & $\begin{array}{l}\text { NAINM, } \\
\text { NA/NM, }\end{array}$ & Yes & $\begin{array}{l}\text { Pons extending } \\
\text { to the upper } \\
\text { cervical cord }\end{array}$ & MHT of the ICA & $\begin{array}{l}\text { A Cerebellar vein and } \\
\text { anterior spinal vein }\end{array}$ & Type V & Open surgery & Completely & 3 months & No & 3 \\
\hline 40 Ko et al. (33) & $54 / \mathrm{M}$ & $\begin{array}{l}\text { Tetrapraresis, } \\
\text { hypesthesia, } \\
\text { diplopia/5 years }\end{array}$ & $\begin{array}{l}\text { Yes (Tolosa-Hunt } \\
\text { syndrome) }\end{array}$ & & No & $\begin{array}{l}\text { NANM, } \\
\text { NANMM }\end{array}$ & Hyper & NA/NM & $\begin{array}{l}\text { NAINM, } \\
\text { NA/NM, }\end{array}$ & Yes & $\begin{array}{l}\text { Medulla } \\
\text { oblongata } \\
\text { extending to the } \\
\text { upper cervical } \\
\text { cord }\end{array}$ & $\begin{array}{l}\text { MHT of the } \\
\text { ICA, MMA }\end{array}$ & $\begin{array}{l}\text { Pontomesencephalic } \\
\text { vein } \rightarrow \text { cervical PMV }\end{array}$ & lifype V & $\begin{array}{l}\text { TAE with NBCA via } \\
\text { multiple feeders }\end{array}$ & a Incompletely & 10 months & $\begin{array}{l}\text { Yes/Second- } \\
\text { stage } \\
\text { embolization } \\
\text { and } \\
\text { gamma-knife } \\
\text { radiosurgery }\end{array}$ & 4 or 5 \\
\hline $\begin{array}{l}41 \text { Kleeberg et al. } \\
\text { (34) }\end{array}$ & $60 / \mathrm{M}$ & $\begin{array}{l}\text { Difficulty to walk/6 } \\
\text { weeks }\end{array}$ & No & Tentorium & No & $\begin{array}{l}\text { NAANM, } \\
\text { NANMM }\end{array}$ & Hyper & NA/NM & $\begin{array}{l}\text { NA/NM, } \\
\text { NAINM }\end{array}$ & Yes & $\begin{array}{l}\text { Medulla } \\
\text { oblongata } \\
\text { extending to the } \\
\text { hypoer cervical } \\
\text { cord }\end{array}$ & $M H T$ of the ICA & $\begin{array}{l}\text { A Cerebellar } \\
\text { vein } \rightarrow \text { PMV }\end{array}$ & Type V & $\begin{array}{l}\text { Combined TAE and } \\
\text { open surgery }\end{array}$ & d Completely & Immediately I & No & 1 or 2 \\
\hline $\begin{array}{l}42 \text { Patsalides et al. } \\
\text { (35) }\end{array}$ & $53 / \mathrm{M}$ & $\begin{array}{l}\text { Syncope attacks } \\
\text { and tingling of the } \\
\text { fingertips } / 3 \text { months }\end{array}$ & Yes (NA/NM) & SPS & Yes & $\begin{array}{l}\text { NANM, } \\
\text { enhanced }\end{array}$ & Hyper & NA/NM & $\begin{array}{l}\text { NAINM, } \\
\text { NAINM }\end{array}$ & & $\begin{array}{l}\text { Medulla } \\
\text { oblongata } \\
\text { extending to the } \\
\text { upper cervical } \\
\text { cord }\end{array}$ & $\begin{array}{l}\text { MHT of the } \\
\text { ICA, MMA }\end{array}$ & $\begin{array}{l}\text { Veins around } \\
\text { brainstem } \rightarrow \text { spinal } \\
\text { veins }\end{array}$ & Type V & $\begin{array}{l}\text { TAE with NBCA via } \\
\text { MHT of the ICA }\end{array}$ & a Completely & 6 months & No & 0 \\
\hline $\begin{array}{l}43 \text { Aixut Lorenzo } \\
\text { et al. (36) }\end{array}$ & $67 / F$ & $\begin{array}{l}\text { Neck pain, } \\
\text { Tetrapraresis, } \\
\text { urinary } \\
\text { retention/several } \\
\text { days }\end{array}$ & No & $\begin{array}{l}\text { Tentorium } \\
\text { (petrosal } \\
\text { ridge) }\end{array}$ & Yes & $\begin{array}{l}\text { NANM, } \\
\text { NANMM }\end{array}$ & Hyper & NA/NM & $\begin{array}{l}\text { NAINM, } \\
\text { NA/NM }\end{array}$ & & $\begin{array}{l}\text { Medulla } \\
\text { oblongata } \\
\text { extending to the } \\
\text { entire cervical } \\
\text { cord }\end{array}$ & $\begin{array}{l}\text { MMA, AphA, } \\
\text { and OA }\end{array}$ & $\begin{array}{l}\text { Vein around } \\
\text { brainstem } \rightarrow \text { spinal } \\
\text { PMV }\end{array}$ & Type V & $\begin{array}{l}\text { TAE with Onyx via } \\
\text { OA }\end{array}$ & Completely & 12 months $Y$ & Yes/TVE & 0 or 1 \\
\hline 44 Kim et al. (37) & $45 / \mathrm{M}$ & $\begin{array}{l}\text { Tetrapraresis and } \\
\text { respiratory } \\
\text { distress/6 months }\end{array}$ & $\begin{array}{l}\text { Yes } \\
\text { (demyelinating } \\
\text { disease) }\end{array}$ & $\begin{array}{l}\text { Tentorium } \\
\text { (petrosal } \\
\text { ridge) }\end{array}$ & No & $\begin{array}{l}\text { NAINM, } \\
\text { enhanced }\end{array}$ & Hyper & Hyper & $\begin{array}{l}\text { Normal, } \\
\text { NA/NM }\end{array}$ & No & $\begin{array}{l}\text { Medulla } \\
\text { oblongata } \\
\text { extending to the } \\
\text { upper cervical } \\
\text { cord }\end{array}$ & $\begin{array}{l}\text { Meningeal } \\
\text { branches of } \\
\text { bilateral ICAs }\end{array}$ & Cervical PMV & Type V & Open surgery & Completely & 2 weeks & No & 3 \\
\hline 45 Peltier et al. (38) & $58 / \mathrm{F}$ & $\begin{array}{l}\text { Tetrapraresis, } \\
\text { urinary retention } \\
\text { and breathing } \\
\text { difficulty/2 months }\end{array}$ & No & $\mathrm{COJ}$ & No & $\begin{array}{l}\text { NANM, } \\
\text { enhanced }\end{array}$ & Hyper & NAINM & $\begin{array}{l}\text { NAINM, } \\
\text { NAINM }\end{array}$ & Yes & $\begin{array}{l}\text { Medulla } \\
\text { oblongata } \\
\text { extending to the } \\
\text { upper cervical } \\
\text { cord }\end{array}$ & PMA of the VA & $\begin{array}{l}C 1 \\
\text { radiculomedullary } \\
\text { vein }\end{array}$ & Type V & $\begin{array}{l}\text { Clipping and } \\
\text { section of the } \\
\text { venous stem }\end{array}$ & Completely & 6 months 1 & No & 3 \\
\hline 46 Clark et al. (39) & 49/F & $\begin{array}{l}\text { Dysarthric with } \\
\text { monotonal } \\
\text { hypophonia and } \\
\text { ataxia/3 months }\end{array}$ & No & cs & No & $\begin{array}{l}\text { NANM, } \\
\text { NANMM }\end{array}$ & Hyper & Hyper & $\begin{array}{l}\text { NA/NM, } \\
\text { NA/NM, }\end{array}$ & Yes & $\begin{array}{l}\text { Pons extending } \\
\text { to the upper } \\
\text { cervical cord }\end{array}$ & $M H T$ of the ICA & ANAINM & NAINM & $\begin{array}{l}\text { TAE to coil the } \\
\text { DAVF }\end{array}$ & Completely & 10 days & No & 2 or 3 \\
\hline $\begin{array}{l}47 \text { Ogbonnaya et al. } \\
(40)\end{array}$ & 64/F & $\begin{array}{l}\text { Paraparesis, } \\
\text { unsteady gait/3 } \\
\text { months }\end{array}$ & No & Tentorium & No & $\begin{array}{l}\text { NA/NM, } \\
\text { non-enhanced }\end{array}$ & Hyper & NA/NM & $\begin{array}{l}\text { NAINM, } \\
\text { NAINM }\end{array}$ & Yes & $\begin{array}{l}\text { Medulla } \\
\text { oblongata } \\
\text { extending to the } \\
\text { upper cervical } \\
\text { cord }\end{array}$ & MMA & PMV & Type V & TAE & Completely & Immediately N & No & 4 or 5 \\
\hline 48 Kulwin et al. (41) & 44/F & $\begin{array}{l}\text { Paraparesis, altered } \\
\text { mental status, } \\
\text { hypopneic/ NA/NM }\end{array}$ & $\begin{array}{l}\text { dYes (brainstem } \\
\text { stroke) }\end{array}$ & SPS & No & $\begin{array}{l}\text { NANM, } \\
\text { enhanced }\end{array}$ & NAINM & Hyper & $\begin{array}{l}\text { NA/NM, } \\
\text { hyper }\end{array}$ & & $\begin{array}{l}\text { Pons and } \\
\text { medulla oblongata }\end{array}$ & $\begin{array}{l}\text { MMA, dural } \\
\text { tabranch of VA }\end{array}$ & $\begin{array}{l}\text { SPS } \rightarrow \text { perimesencep } \\
\text { vein } \rightarrow \text { PMV }\end{array}$ & epryabiact & $\begin{array}{l}\text { Surgical } \\
\text { disconnection by } \\
\text { clipping draining } \\
\text { vein }\end{array}$ & Completely & Immediately I & No & 4 or 5 \\
\hline
\end{tabular}


TABLE 1 | Continued

\begin{tabular}{|c|c|c|c|c|c|c|c|c|c|c|c|c|c|c|c|c|c|c|c|}
\hline \multirow{3}{*}{$\begin{array}{l}\text { No.Author/year } \\
49 \text { Clark et al. (42) }\end{array}$} & \multirow{3}{*}{$\begin{array}{c}\text { Age/sex } \\
65 / F \\
\end{array}$} & \multirow{3}{*}{$\begin{array}{l}\begin{array}{l}\text { Presentation/ } \\
\text { interval to } \\
\text { definite diagnosis }\end{array} \\
\begin{array}{l}\text { Tetrapraresis, } \\
\text { gastroenteritis, } \\
\text { urinary } \\
\text { retention/several } \\
\text { days }\end{array} \\
\end{array}$} & \multirow{3}{*}{$\begin{array}{l}\begin{array}{l}\text { Initial } \\
\text { misdiagnosis }\end{array} \\
\text { No }\end{array}$} & \multirow{3}{*}{$\begin{array}{l}\text { DAVF } \\
\text { location }\end{array}$} & \multirow{3}{*}{$\begin{array}{l}\text { Concurrent with } \\
\text { venous sinus } \\
\text { stenosis/ } \\
\text { occlusion }\end{array}$} & \multicolumn{5}{|c|}{ Signal alteration on MRI } & \multirow{2}{*}{$\begin{array}{l}\text { Region of } \\
\text { congestion } \\
\text { l }\end{array}$} & \multirow{2}{*}{$\begin{array}{l}\text { Feeding } \\
\text { artery }\end{array}$} & \multirow[t]{2}{*}{ Draining vein } & \multirow{2}{*}{$\begin{array}{l}\text { Cognard } \\
\text { classificatio }\end{array}$} & \multirow{2}{*}{$\begin{array}{l}\text { Treatment } \\
\text { ion }\end{array}$} & \multirow{2}{*}{$\begin{array}{l}\text { Degree of } \\
\text { DAVF } \\
\text { obliteration }\end{array}$} & \multirow{2}{*}{$\begin{array}{l}\text { Follow-up } \\
\text { period }\end{array}$} & \multirow{2}{*}{ Retreatment } & \multirow{2}{*}{$\begin{array}{l}\text { Outcome } \\
\text { (mRS) }\end{array}$} \\
\hline & & & & & & $\mathrm{T} 1, \mathrm{~T} 1 \mathrm{C}_{+}$ & T2 & FLAIR & $\begin{array}{l}\text { DWI, } \\
\text { ADC }\end{array}$ & $\begin{array}{l}\text { Abnormal } \\
\text { vascular } \\
\text { flow-void }\end{array}$ & & & & & & & & & \\
\hline & & & & & & $\begin{array}{l}\text { NA/NM, } \\
\text { NA/NM }\end{array}$ & Hyper & NA/NM & $\begin{array}{l}\text { NANM, } \\
\text { NANMM }\end{array}$ & No & $\begin{array}{l}\text { Medulla } \\
\text { oblongata and } \\
\text { upper cervical } \\
\text { spinal cord }\end{array}$ & $\begin{array}{l}\text { MMA, MHT of } \\
\text { the ICA }\end{array}$ & $\begin{array}{l}\text { veins around } \\
\text { brainstem } \rightarrow \text { PMV }\end{array}$ & Type V & \multicolumn{2}{|c|}{$\begin{array}{l}\text { Combined TAE and Completely } \\
\text { surgical obliteration }\end{array}$} & Immediately & No & NAINM \\
\hline $\begin{array}{l}50 \text { Mathon et al. } \\
(43)\end{array}$ & $60 / F$ & $\begin{array}{l}\text { Progressive } \\
\text { ascending } \\
\text { myelopathy } \\
\text { associated with } \\
\text { autonomic } \\
\text { dysfunction/NANM }\end{array}$ & No & SPS & No & $\begin{array}{l}\text { NA/NM, } \\
\text { NA/NM }\end{array}$ & Hyper & Hyper & $\begin{array}{l}\text { NANM, } \\
\text { NANMM }\end{array}$ & Yes & $\begin{array}{l}\text { medulla } \\
\text { oblongata with } \\
\text { cervical spinal } \\
\text { cord, }\end{array}$ & $\begin{array}{l}\text { Meningeal } \\
\text { arteries of the } \\
\text { posterior } \\
\text { surface of the } \\
\text { internal carotid } \\
\text { artery, MMA }\end{array}$ & $\begin{array}{l}\text { Dilated } \\
\text { perimedullary veins. }\end{array}$ & Type V & $\begin{array}{l}\text { TAE with glue via } \\
\text { MMA }\end{array}$ & Completely & 1 month & No & 0 \\
\hline $\begin{array}{l}51 \text { Salamon et al. } \\
\text { (44) }\end{array}$ & $43 / \mathrm{M}$ & $\begin{array}{l}\text { Paraparesis, urinary) } \\
\text { retention, vomititing, } \\
\text { hiccups/NANMM }\end{array}$ & & $\begin{array}{l}\text { CCJ } \\
\text { (foramen } \\
\text { magnum) }\end{array}$ & No & $\begin{array}{l}\text { NA/NM, } \\
\text { NA/NM }\end{array}$ & Hyper & NAINM & $\begin{array}{l}\text { NANM, } \\
\text { NANMM }\end{array}$ & Yes & $\begin{array}{l}\text { Hypoer } \\
\text { medulla oblongatab } \\
\text { extending to the } \\
\text { upper cervical } \\
\text { cord }\end{array}$ & $\begin{array}{l}\text { Meningeal } \\
\text { abranch from the } \\
\text { VA }\end{array}$ & $\begin{array}{l}\text { Cerebellar } \\
\text { eveins } \rightarrow \text { venous } \\
\text { drainage along the } \\
\text { medulla } \rightarrow \text { PMV }\end{array}$ & Type V & TAE with Onyx & Completely & 3 months & No & 0 \\
\hline 52 Singh et al. (45) & $\begin{array}{l}\text { Middle- } \\
\text { aged/M }\end{array}$ & $\begin{array}{l}\text { Paraparesis, } \\
\text { urinary } \\
\text { retention, } \\
\text { vomiting, } \\
\text { hiccups/4 months }\end{array}$ & $\begin{array}{l}\text { Yes (periodic } \\
\text { paralysis) }\end{array}$ & Tentorium & No & $\begin{array}{l}\text { NA/NM, } \\
\text { NA/NM }\end{array}$ & Hyper & Hyper & $\begin{array}{l}\text { NA/NM, } \\
\text { NA/NMM }\end{array}$ & Yes & $\begin{array}{l}\text { Pons, medulla } \\
\text { oblongata } \\
\text { extending to the } \\
\text { upper cervical } \\
\text { cord }\end{array}$ & $\begin{array}{l}\text { MHAs of the } \\
\text { ICAs, MMA }\end{array}$ & $\begin{array}{l}\text { Perimesencephalic } \\
\text { vein and PMV }\end{array}$ & Type V & Open surgery & Completely & 3 months & No & 0 \\
\hline 53 El Asri et al. (46) & $48 / \mathrm{M}$ & $\begin{array}{l}\text { Tetrapraresis, } \\
\text { hypaesthesia, } \\
\text { breathing } \\
\text { dificiculty/10 days }\end{array}$ & No & Tentorium & No & $\begin{array}{l}\text { NA/NM, } \\
\text { non-enhanced }\end{array}$ & Hyper & NA/NM & $\begin{array}{l}\text { NANM, } \\
\text { NA/NM }\end{array}$ & Yes & $\begin{array}{l}\text { Hypoer medulla } \\
\text { oblongata } \\
\text { extending to the } \\
\text { upper cervical } \\
\text { cord }\end{array}$ & MHT of the ICA & $\begin{array}{l}\text { A Cerebellar } \\
\text { veins } \rightarrow \text { PMV }\end{array}$ & Type V & Open surgery & Completely & 2 years & No & 4 or 5 \\
\hline $\begin{array}{l}54 \text { Foreman et al. } \\
\text { (47) }\end{array}$ & $59 / F$ & $\begin{array}{l}\text { Tetrapraresis, pain, Y } \\
\text { urinary retention/3 o } \\
\text { weeks }\end{array}$ & $\begin{array}{l}\text { Yes (infarction or } \\
\text { contusion) }\end{array}$ & $r \cos$ & No & $\begin{array}{l}\text { NA/NM, } \\
\text { non-enhanced }\end{array}$ & Hyper & NA/NM & $\begin{array}{l}\text { NANM, } \\
\text { NANMM }\end{array}$ & Yes & $\begin{array}{l}\text { Medulla oblongatal } \\
\text { and entire cervical } \\
\text { spinal cord }\end{array}$ & aMHT of the ICA & $\begin{array}{l}\text { A Pontomesencephali } \\
\text { vein } \rightarrow \text { PMV }\end{array}$ & lifype V & Open surgery & Completely & Immediately & No & 4 or 5 \\
\hline 55 Gross et al. (48) & 69/M & $\begin{array}{l}\text { Progressive } \\
\text { hypoer extremity } \\
\text { weakness and } \\
\text { urinary retention/3 } \\
\text { days }\end{array}$ & $\begin{array}{l}\text { Yes } \\
\text { (Guilian-Barre } \\
\text { syndrome) }\end{array}$ & Tentorium & No & $\begin{array}{l}\text { NAANM, } \\
\text { NAANM }\end{array}$ & Hyper & Hyper & $\begin{array}{l}\text { NA/NM, } \\
\text { NA/NM }\end{array}$ & Yes & $\begin{array}{l}\text { Pons, medulla, } \\
\text { and upper } \\
\text { cervical spine }\end{array}$ & $\begin{array}{l}\text { MMA, tentorial } \\
\text { branch of ICA, } \\
\text { dural branches } \\
\text { of OA and } \\
\text { posterior } \\
\text { auricular artery }\end{array}$ & $\begin{array}{l}\text { Cervical spinal } \\
\text { veins }\end{array}$ & Type V & TAE with Onyx & Completely & 10 weeks & No & 3 \\
\hline 56 & $34 / F$ & 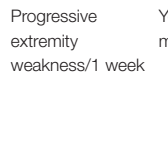 & $\begin{array}{l}\text { Yes (transverse } \\
\text { myelitis) }\end{array}$ & TS-SS & No & $\begin{array}{l}\text { NA/NM, } \\
\text { NA/NM }\end{array}$ & Hyper & Hyper & $\begin{array}{l}\text { NANM, } \\
\text { NANMM }\end{array}$ & Yes & $\begin{array}{l}\text { Brainstem and } \\
\text { cervicomedullary } \\
\text { junction }\end{array}$ & $\mathrm{OA}$ & $\begin{array}{l}\text { SPS } \rightarrow \text { petrosal vein } \\
\text { and medullary } \\
\text { vein } \rightarrow \text { anterior } \\
\text { spinal vein and } \\
\text { cervicomedullary } \\
\text { vein }\end{array}$ & Type V & TAE with Onyx & Completely & 3 months & No & 0 \\
\hline 57 Wu et al. (49) & 46/F & $\begin{array}{l}\text { Paraparesis, } \\
\text { vertigo, vomiting } \\
\text { and dysphagia/1 } \\
\text { month }\end{array}$ & $\begin{array}{l}\text { Yes (brainstem } \\
\text { infarction) }\end{array}$ & cos & No & $\begin{array}{l}\text { NA/NM, partial } \\
\text { enhancement }\end{array}$ & Hyper & Hyper & $\begin{array}{l}\text { NANM, } \\
\text { NANMM }\end{array}$ & Yes & $\begin{array}{l}\text { Pons, medulla } \\
\text { oblongata. } \\
\end{array}$ & $\begin{array}{l}\text { Meningeal } \\
\text { branch from the } \\
\text { radicular artery } \\
\text { of the VA }\end{array}$ & $\begin{array}{l}\text { Pontomesencephalid } \\
\text { eveins } \rightarrow \text { basal vein } \\
\text { 'and anterior spinal } \\
\text { vein }\end{array}$ & lifype V & TAE with Onyx & Completely & 6 months & No & 0 \\
\hline 58 Haryu et al. (50) & $62 / \mathrm{M}$ & $\begin{array}{l}\text { Upper limb } \\
\text { weakness and } \\
\text { diffifulty in } \\
\text { walking/4 months }\end{array}$ & NAINM & $\begin{array}{l}\text { Tentorium } \\
\text { (petrosal } \\
\text { ridge) }\end{array}$ & NAINM & $\begin{array}{l}\text { NANM, } \\
\text { NANMM }\end{array}$ & Hyper & NAINM & $\begin{array}{l}\text { NANM, } \\
\text { NANMM }\end{array}$ & Yes & $\begin{array}{l}\text { Cervical spinal } \\
\text { cord and medulla } \\
\text { oblongata }\end{array}$ & MMA & $\begin{array}{l}\text { Petrosal vein into } \\
\text { the anterior spinal } \\
\text { veins }\end{array}$ & Type V & Open surgery & Completely & 18 months & No & 2 \\
\hline 59 & $64 / \mathrm{M}$ & $\begin{array}{l}\text { Myelopathy, bulbar Y } \\
\text { palsy/NANM }\end{array}$ & Yes (NA/NM) & NAINM & NAINM & $\begin{array}{l}\text { NA/NM, } \\
\text { heterogeneousl, } \\
\text { enhanced }\end{array}$ & $\begin{array}{l}\text { Hyper } \\
\text { sly }\end{array}$ & NA/NM & $\begin{array}{l}\text { NA/NM, } \\
\text { NA/NM }\end{array}$ & Yes & $\begin{array}{l}\text { Cervical spinal } \\
\text { cord and medulla } \\
\text { oblongata }\end{array}$ & NAINM & Spinal veins & Type V & NA/NM & NA/NM & NA/NM & NANM & 3 \\
\hline
\end{tabular}




\begin{tabular}{|c|c|c|c|c|c|c|c|c|c|c|c|c|c|c|c|c|c|c|c|}
\hline \multirow[t]{2}{*}{ No.Author/year } & \multirow[t]{2}{*}{ Age/sex } & \multirow{2}{*}{$\begin{array}{l}\text { Presentation/ } \\
\text { interval to } \\
\text { definite diagnosis }\end{array}$} & \multirow{2}{*}{$\begin{array}{l}\text { Initial } \\
\text { misdiagnosis }\end{array}$} & \multirow[t]{2}{*}{$\begin{array}{l}\text { DAVF } \\
\text { location }\end{array}$} & \multirow{2}{*}{$\begin{array}{l}\text { Concurrent with } \\
\text { venous sinus } \\
\text { stenosis/ } \\
\text { occlusion }\end{array}$} & \multicolumn{5}{|c|}{ Signal alteration on MRI } & \multirow{2}{*}{$\begin{array}{l}\text { Region of } \\
\text { congestion } \\
\text { al } \\
\text { id }\end{array}$} & \multirow[t]{2}{*}{$\begin{array}{l}\text { Feeding } \\
\text { artery }\end{array}$} & \multirow[t]{2}{*}{ Draining vein } & \multirow[t]{2}{*}{$\begin{array}{l}\text { Cognard } \\
\text { classificatio }\end{array}$} & \multirow[t]{2}{*}{$\begin{array}{l}\text { Treatment } \\
\text { on }\end{array}$} & \multirow{2}{*}{$\begin{array}{l}\text { Degree of } \\
\text { DAVF } \\
\text { obliteration }\end{array}$} & \multirow[t]{2}{*}{$\begin{array}{l}\text { Follow-up } \\
\text { period }\end{array}$} & \multirow[t]{2}{*}{ Retreatment } & \multirow[t]{2}{*}{$\begin{array}{l}\text { Outcome } \\
\text { (mPS) }\end{array}$} \\
\hline & & & & & & $\mathrm{T} 1, \mathrm{~T} 1 \mathrm{C}_{+}$ & T2 & FLAIR & $\begin{array}{l}\text { DWI, } \\
\text { ADC }\end{array}$ & $\begin{array}{l}\text { Abnormal } \\
\text { vascular } \\
\text { flow-void }\end{array}$ & & & & & & & & & \\
\hline 60 & 68/M & $\begin{array}{l}\text { Myelopathy, } \\
\text { respiratory } \\
\text { failure/NANM }\end{array}$ & NAINM & NANM & NAINM & $\begin{array}{l}\text { NA/NM, } \\
\text { non-enhanced }\end{array}$ & Hyper & NA/NM & $\begin{array}{l}\text { NA/NM, } \\
\text { NA/NM }\end{array}$ & Yes & $\begin{array}{l}\text { Cervical spinal } \\
\text { cord and medulla } \\
\text { oblongata }\end{array}$ & AphA & Anterior spinal veins & SType $V$ & Open surgery & NANM & NANM & NANM & 4 \\
\hline 61 Roelz et al. (51) & 76/M & $\begin{array}{l}\text { Nausea and } \\
\text { vomiting, inability tog } \\
\text { wakl, and blurred } \\
\text { vision/8 months }\end{array}$ & $\begin{array}{l}\text { Yes (brainstem } \\
\text { oglioma or } \\
\text { lymphoma) }\end{array}$ & $\begin{array}{l}\text { CCJ } \\
\text { (Posterior } \\
\text { jugular } \\
\text { foramen) }\end{array}$ & No & $\begin{array}{l}\text { NA/NM, } \\
\text { enhanced }\end{array}$ & Hyper & Hyper & $\begin{array}{l}\text { NA/NM, } \\
\text { NA/NM }\end{array}$ & Yes & $\begin{array}{l}\text { Pontomedullary } \\
\text { junction } \\
\text { extending to } \\
\text { inferior cerebellar } \\
\text { peduncle }\end{array}$ & $\begin{array}{l}\text { MMA, AphA, } \\
\text { PAA, and OA }\end{array}$ & $\begin{array}{l}\text { Lateral medullary } \\
\text { into the anterior } \\
\text { perimedulary/perisp } \\
\text { veins }\end{array}$ & $\begin{array}{l}\text { Type V } \\
\text { oinal }\end{array}$ & $\begin{array}{l}\text { TAE with Onyx via } \\
\text { MMA, AphA, PAA }\end{array}$ & Completely & 10 months & $\begin{array}{l}\text { Yes/combined2 } 2 \\
\text { endovascular } \\
\text { (via OA) and } \\
\text { surgical } \\
\text { approach }\end{array}$ & \\
\hline 62 Le et al. (52) & $36 / \mathrm{M}$ & $\begin{array}{l}\text { Headache, } \\
\text { hypoesthesia, } \\
\text { vomititg, ataxia/2 } \\
\text { months }\end{array}$ & $\begin{array}{l}\text { Yes (brainstem } \\
\text { glioma) }\end{array}$ & $\begin{array}{l}\text { Tentorium } \\
\text { (petrosal } \\
\text { apex) }\end{array}$ & No & $\begin{array}{l}\text { Normal, } \\
\text { punctiform } \\
\text { enhancement }\end{array}$ & Hyper & Hyper & $\begin{array}{l}\text { NA/NM, } \\
\text { NA/NM }\end{array}$ & Yes & $\begin{array}{l}\text { Medulla } \\
\text { oblongata }\end{array}$ & $\begin{array}{l}\text { MMAs, AphA, } \\
\text { internal } \\
\text { maxillary artery }\end{array}$ & Spinal PMV & Type V & TAE with NBCA & Completely & 1 year & No & 0 \\
\hline 63 Alvare et al. (53) & 69/M & $\begin{array}{l}\text { Nausea, vomiting, }{ }^{Y} \\
\text { paraparesis/NANM }\end{array}$ & Yes (encephalitis) & $\begin{array}{l}\text { Tentorium } \\
\text { (petrosal } \\
\text { ridge) }\end{array}$ & No & $\begin{array}{l}\text { NA/NM, } \\
\text { enhanced }\end{array}$ & Hyper & Hyper & $\begin{array}{l}\text { Normal, } \\
\text { hyper }\end{array}$ & No & $\begin{array}{l}\text { Pons and } \\
\text { medulla oblongata }\end{array}$ & $\begin{array}{l}\text { MMA, anterior } \\
\text { ainferior } \\
\text { cerebellar artery }\end{array}$ & $\begin{array}{l}\text { Veins of the } \\
\text { cerebello-pontine } \\
\text { yangle } \rightarrow \text { veins } \\
\text { around the } \\
\text { brainstem } \rightarrow \text { spinal } \\
\text { PMV }\end{array}$ & Type V & $\begin{array}{l}\text { Combined TAE and } \\
\text { clip and coagulate } \\
\text { the draining vein }\end{array}$ & Completely & 3 months & No & 0 \\
\hline 64 Pop et al. (54) & 38/M & $\begin{array}{l}\text { Seizure, tetraplegia, } \\
\text { respiritary } \\
\text { difficityly/1 month }\end{array}$ & $\begin{array}{l}\text { a, Yes } \\
\text { (Guillain-Barre } \\
\text { syndrome) }\end{array}$ & $\begin{array}{l}\text { CCJ } \\
\text { (foramen } \\
\text { magnum) }\end{array}$ & No & $\begin{array}{l}\text { Low, } \\
\text { non-enhanced }\end{array}$ & Hyper & Hyper & $\begin{array}{l}\text { NAANM, } \\
\text { NA/NM }\end{array}$ & Yes & $\begin{array}{l}\text { Medulla } \\
\text { oblongata } \\
\text { extending to the } \\
\text { entire cervical } \\
\text { cord }\end{array}$ & $\mathrm{OA}, \mathrm{AphA}$ & $\begin{array}{l}\text { Bidirectional } \\
\text { drainage to cortical } \\
\text { temporal vein and } \\
\text { spinal veins }\end{array}$ & Type V & $\begin{array}{l}\text { TAE with Onyx via } \\
\text { OA }\end{array}$ & Completely & 6 months & No & 3 \\
\hline 65 Abud et al. (55) & $66 / F$ & $\begin{array}{l}\text { Tetraparesis/1 } \\
\text { month }\end{array}$ & No & ss & No & $\begin{array}{l}\text { NA/NM, } \\
\text { non-enhanced }\end{array}$ & Hyper & NA/NM & $\begin{array}{l}\text { NA/NM, } \\
\text { NA/NM }\end{array}$ & Yes & $\begin{array}{l}\text { Medulla } \\
\text { oblongata } \\
\text { extending to the } \\
\text { upper cervical } \\
\text { cord }\end{array}$ & $\mathrm{OA}$ & $\begin{array}{l}\text { Cerebellar cortical } \\
\text { venous } \\
\text { drainage } \rightarrow \text { PMV }\end{array}$ & Type V & $\begin{array}{l}\text { TAE with Onyx via } \\
\text { OA }\end{array}$ & Completely & 3 months & No & 0 \\
\hline $\begin{array}{l}66 \text { Abdelsadg et al. } \\
\text { (56) }\end{array}$ & 65/F & $\begin{array}{l}\text { Tetraparesis, } \\
\text { dizziness, urination } \\
\text { dificulty/several } \\
\text { days }\end{array}$ & No & $\cos$ & Yes & $\begin{array}{l}\text { NA/NM, } \\
\text { NA/NM }\end{array}$ & Hyper & Hyper & $\begin{array}{l}\text { NA/NM, I } \\
\text { Hyper }\end{array}$ & No & $\begin{array}{l}\text { Medulla } \\
\text { oblongata } \\
\text { extending to the } \\
\text { upper cervical } \\
\text { cord }\end{array}$ & $\begin{array}{l}\text { MHT of the } \\
\text { ICA, MMA, }\end{array}$ & $\begin{array}{l}\text { SPS } \rightarrow \text { brainstem } \\
\text { and cervical PMV }\end{array}$ & Type V & TAE & Completely & 3 months & No & 3 \\
\hline $\begin{array}{l}67 \text { Enokizono et al. } \\
\text { (57) }\end{array}$ & $50 \mathrm{~s} / \mathrm{F}$ & $\begin{array}{l}\text { Tetraparesis, } \\
\text { numbness of limbs, } \\
\text { urination difficulty/1 } \\
\text { month }\end{array}$ & No & $\mathrm{coJ}$ & No & $\begin{array}{l}\text { NA/NM, } \\
\text { NA/NM }\end{array}$ & Hyper & NAINM & $\begin{array}{l}\text { NA/NM, } \\
\text { NA/NM }\end{array}$ & Yes & $\begin{array}{l}\text { Medulla } \\
\text { oblongata } \\
\text { extending to the } \\
\text { upper thoracic } \\
\text { cord }\end{array}$ & $\begin{array}{l}\text { Meningeal } \\
\text { branch from the } \\
\text { radicular artery } \\
\text { of the VA }\end{array}$ & $\begin{array}{l}\text { Anterior and } \\
\text { eposterior spinal } \\
\text { veins }\end{array}$ & Type V & Open surgery & Completely & NANM & No & NA/NM \\
\hline 68 & $60 \mathrm{~s} / \mathrm{M}$ & $\begin{array}{l}\text { Tetraparesis, } \\
\text { numbness of limbs, } \\
\text { urination difficulty/7 } \\
\text { months }\end{array}$ & No & Tentorium & No & $\begin{array}{l}\text { NA/NM, } \\
\text { NA/NM }\end{array}$ & Hyper & NAINM & $\begin{array}{l}\text { NA/NM, } \\
\text { NA/NM }\end{array}$ & Yes & $\begin{array}{l}\text { Medulla } \\
\text { oblongata } \\
\text { extending to the } \\
\text { upper thoracic } \\
\text { cord }\end{array}$ & $\begin{array}{l}\text { MHT of the } \\
\text { ICA, MMA, } \\
\text { accessory } \\
\text { meningeal } \\
\text { artery }\end{array}$ & $\begin{array}{l}\text { Petrosal } \\
\text { vein } \rightarrow \text { veins around } \\
\text { the } \\
\text { brainstem } \rightarrow \text { PMV }\end{array}$ & Type V & Open surgery & Completely & NAINM & No & NAINM \\
\hline 69 & $60 \mathrm{~s} / \mathrm{M}$ & $\begin{array}{l}\text { Tetraparesis, } \\
\text { numbess of limbs, } \\
\text { respiratory } \\
\text { difficulty/2 months }\end{array}$ & No & Tentorium & No & $\begin{array}{l}\text { NA/NM, } \\
\text { NANMM }\end{array}$ & Hyper & NAINM & $\begin{array}{l}\text { NAANM, } \\
\text { NA/NM }\end{array}$ & Yes & $\begin{array}{l}\text { Medulla } \\
\text { oblongata } \\
\text { extending to the } \\
\text { entire cenvical } \\
\text { cord }\end{array}$ & MMA & $\begin{array}{l}\text { Petrosal } \\
\text { vein } \rightarrow \text { veins around } \\
\text { the } \\
\text { brainstem } \rightarrow \text { PMV }\end{array}$ & Type V & $\begin{array}{l}\text { Combined TAE and } \\
\text { clip the draining } \\
\text { vein }\end{array}$ & d Completely & NAINM & No & NA/NM \\
\hline 70 Tanaka et al. (58) & $64 / \mathrm{M}$ & $\begin{array}{l}\text { Paraparesis, } \\
\text { bladder } \\
\text { dysfunction/NANM }\end{array}$ & No & $\begin{array}{l}\text { Occipital } \\
\text { sinus }\end{array}$ & Yes & $\begin{array}{l}\text { NA/M, } \\
\text { NANMM }\end{array}$ & Hyper & NA/NM & $\begin{array}{l}\text { NA/MM, I } \\
\text { NA/NM }\end{array}$ & № & $\begin{array}{l}\text { Medulla } \\
\text { oblongata } \\
\text { extending to the } \\
\text { upper cervical } \\
\text { cord }\end{array}$ & $\begin{array}{l}\text { PMAs of the } \\
\text { VAs }\end{array}$ & $\begin{array}{l}\text { Occipital } \\
\text { sinus } \rightarrow \text { anterior } \\
\text { spinal vein }\end{array}$ & Type V & $\begin{array}{l}\text { TAE with Onyx via } \\
\text { PAAs of the VAs }\end{array}$ & Completely & 8 months & No & 2 \\
\hline 71 Emmer et al. (59) & $65 / \mathrm{M}$ & $\begin{array}{l}\text { Eye movement } \\
\text { abnormalities, limb } \\
\text { weakness, and gait } \\
\text { instability/2 years }\end{array}$ & Yes (tumor) & $\mathrm{COJ}$ & No & $\begin{array}{l}\text { NA/NM, } \\
\text { heterogeneously } \\
\text { enhanced }\end{array}$ & $\begin{array}{l}\text { Hyper } \\
\text { y }\end{array}$ & Hyper & $\begin{array}{l}\text { NAANM, } \\
\text { NA/NM }\end{array}$ & Yes & $\begin{array}{l}\text { Medulla } \\
\text { oblongata and } \\
\text { cerebellum }\end{array}$ & PMA of the VA & Cerebellar vein & Type III & $\begin{array}{l}\text { TAE with NBCA via } \\
\text { PMA }\end{array}$ & Completely & Immediately I & No & 4 or 5 \\
\hline 72 Duan et al. (60) & 67/F & $\begin{array}{l}\text { Paraparesis, } \\
\text { headache and } \\
\text { progressive } \\
\text { Confusion/1 month }\end{array}$ & $\begin{array}{l}\text { Yes (brainstem } \\
\text { tumor) }\end{array}$ & SPS & No & $\begin{array}{l}\text { NANM, } \\
\text { partially } \\
\text { enhanced }\end{array}$ & Hyper & Hyper & $\begin{array}{l}\text { Hyper, } \\
\text { NAAMM }\end{array}$ & No & $\begin{array}{l}\text { Cerebellum and } \\
\text { pons }\end{array}$ & $M M A, O A$ & $\mathrm{SPS} \rightarrow \mathrm{PMV}$ & Type V & TAE & Completely & Immediately I & No & 4 or 5 \\
\hline
\end{tabular}


TABLE 1 | Continued

\begin{tabular}{|c|c|c|c|c|c|c|c|c|c|c|c|c|c|c|c|c|c|c|c|}
\hline \multirow{3}{*}{$\begin{array}{l}\text { No.Author/year } \\
73 \text { Chen et al. (61) }\end{array}$} & \multirow{3}{*}{$\begin{array}{r}\text { Age/sex } \\
\\
25 / \mathrm{F}\end{array}$} & \multirow{3}{*}{$\begin{array}{l}\text { Presentation/ } \\
\text { interval to } \\
\text { definite diagnosis }\end{array}$} & \multirow{3}{*}{$\begin{array}{l}\text { Initial } \\
\text { misdiagnosis }\end{array}$} & \multirow{3}{*}{$\begin{array}{l}\text { DAVF } \\
\text { location } \\
\text { Posterior } \\
\text { fossa }\end{array}$} & \multirow{3}{*}{ 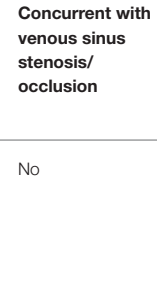 } & \multicolumn{5}{|c|}{ Signal alteration on MRI } & \multirow{2}{*}{$\begin{array}{l}\text { Region of } \\
\text { congestion } \\
\text { al } \\
\text { id } \\
\text { id }\end{array}$} & \multirow{2}{*}{$\begin{array}{l}\text { Feeding } \\
\text { artery }\end{array}$} & \multirow[t]{2}{*}{ Draining vein } & \multirow{2}{*}{$\begin{array}{l}\text { Cognard } \\
\text { classificatio }\end{array}$} & \multirow{2}{*}{$\begin{array}{l}\text { Treatment } \\
\text { ion }\end{array}$} & \multirow{2}{*}{$\begin{array}{l}\text { Degree of } \\
\text { DAVF } \\
\text { obliteration }\end{array}$} & \multirow{2}{*}{$\begin{array}{l}\text { Follow-up } \\
\text { period }\end{array}$} & \multirow{2}{*}{ Retreatment } & \multirow{2}{*}{$\begin{array}{l}\text { t Outcome } \\
\text { (mRS) }\end{array}$} \\
\hline & & & & & & $\mathrm{T} 1, \mathrm{~T} 1 \mathrm{C}+$ & T2 & FLAIR & $\begin{array}{l}\text { DWI, } \\
\text { ADC }\end{array}$ & $\begin{array}{l}\text { Abnormal } \\
\text { vascular } \\
\text { flow-void }\end{array}$ & & & & & & & & & \\
\hline & & & & & & $\begin{array}{l}\text { NA/NM, } \\
\text { enhanced }\end{array}$ & Hyper & NAINM & $\begin{array}{l}\text { NA/NM, } \\
\text { NA/NM }\end{array}$ & , Yes & Pons to C2/C3 & $\begin{array}{l}\text { Posterior } \\
\text { meningeal } \\
\text { branch of VA }\end{array}$ & Anterior spinal vein & Type V & TAE & Completely & NANM & No & NANM \\
\hline $\begin{array}{l}74 \text { Bernard et al. } \\
\text { (62) }\end{array}$ & $65 / \mathrm{M}$ & $\begin{array}{l}\text { Progressive ataxia, } \\
\text { swalhypoing dnorma } \\
\text { and bilateral } \\
\text { tinnitus/5 months }\end{array}$ & $\begin{array}{l}\text { Yes (glioma) } \\
\text { nalrders, }\end{array}$ & CCJ & No & $\begin{array}{l}\text { NA/NM, } \\
\text { enhanced }\end{array}$ & Hyper & NAINM & $\begin{array}{l}\text { NA/NM, } \\
\text { NA/NM }\end{array}$ & , Yes & $\begin{array}{l}\text { Medulla and } \\
\text { hyper cervical } \\
\text { cord }\end{array}$ & $\begin{array}{l}\text { Branches of } \\
\text { AphA }\end{array}$ & $\begin{array}{l}\text { Cerebellar } \\
\text { medullary vein } \\
\text { (white arrowhead) } \\
\text { reaching } \\
\text { perimedullary veins }\end{array}$ & Type V & Open surgery & Completely & 1 month & No & 0 \\
\hline 75 Zhang et al. (4) & $33 / \mathrm{M}$ & $\begin{array}{l}\text { Progressive } \\
\text { weakness of the } \\
\text { hypoer extremities } \\
\text { and gait } \\
\text { disturbance/2 } \\
\text { months }\end{array}$ & $\begin{array}{l}\text { Yes (transverse } \\
\text { myelitis) }\end{array}$ & Tentorium & No & $\begin{array}{l}\text { NA/NM, patchy } \\
\text { enhancement }\end{array}$ & ny Hyper & NAINM & $\begin{array}{l}\text { NA/NM, } \\
\text { NA/NM }\end{array}$ & , Yes & $\begin{array}{l}\text { Medulla } \\
\text { oblongata and } \\
\text { cervical spinal } \\
\text { cord }\end{array}$ & MHT of ICA & Perimedullary veins & S Type V & TAE with Onyx & Completely & 1 month & No & 2 \\
\hline 76 Li et al. (63) & $54 / \mathrm{F}$ & $\begin{array}{l}\text { Limb weakness } \\
\text { and sphincter } \\
\text { dysunction/20 } \\
\text { days }\end{array}$ & No & $\begin{array}{l}\text { Tentorium } \\
\text { (petrosal } \\
\text { apex) }\end{array}$ & No & $\begin{array}{l}\text { NA/NM, non- } \\
\text { enhanced }\end{array}$ & Hyper & NANM & $\begin{array}{l}\text { NA/NM, } \\
\text { NA/NM }\end{array}$ & , Yes & $\begin{array}{l}\text { Medulla } \\
\text { oblongata and } \\
\text { cervical spinal } \\
\text { cord }\end{array}$ & MHT of ICA & $\begin{array}{l}\text { Medullary into the } \\
\text { perimedullary }\end{array}$ & Type V & Open surgery & Completely & 1 month & No & 1 \\
\hline 77 Wang et al. (64) & $53 / \mathrm{M}$ & $\begin{array}{l}\text { Numbness of the } \\
\text { limbs, gait } \\
\text { disturbance and } \\
\text { cough/NA/NM }\end{array}$ & No & $\mathrm{cos}$ & No & $\begin{array}{l}\text { NA/NM, patchy } \\
\text { enhancement }\end{array}$ & ny Hyper & Hyper & $\begin{array}{l}\text { Hyper, } \\
\text { NAINM }\end{array}$ & Yes & $\begin{array}{l}\text { Pons to medulla } \\
\text { oblongata }\end{array}$ & OA of the VA & $\begin{array}{l}\text { SS and cortical } \\
\text { venous drainage }\end{array}$ & Type IIB & $\begin{array}{l}\text { TAE with Onyx via } \\
\text { OA }\end{array}$ & Completely & Immediately I & No & 3 \\
\hline 78 & $53 / \mathrm{M}$ & $\begin{array}{l}\text { Tetrapraresis, } \\
\text { hypaesthesia, } \\
\text { swahlyyoing } \\
\text { difficulty/2 months }\end{array}$ & No & CCJ & No & $\begin{array}{l}\text { Low, non- } \\
\text { enhanced }\end{array}$ & Hyper & Hyper & $\begin{array}{l}\text { Normal, } \\
\text { NA/NM }\end{array}$ & , Yes & $\begin{array}{l}\text { Medulla } \\
\text { oblongata }\end{array}$ & $\begin{array}{l}\text { Dural branch of } \\
\text { VA }\end{array}$ & $\begin{array}{l}\text { f Posterior spinal } \\
\text { veins }\end{array}$ & Type V & $\begin{array}{l}\text { Coagulating and } \\
\text { cutting draining } \\
\text { veins }\end{array}$ & Completely & Immediately I & No & 4 or 5 \\
\hline $\begin{array}{l}79 \text { Takahashi et al. } \\
\text { (65) }\end{array}$ & $63 / \mathrm{M}$ & $\begin{array}{l}\text { Tetraparesis, } \\
\text { respiratory falure/5 } \\
\text { months }\end{array}$ & $5^{\text {No }}$ & $\mathrm{COJ}$ & No & $\begin{array}{l}\text { NA/NM, } \\
\text { NAINM }\end{array}$ & Hyper & NAINM & $\begin{array}{l}\text { NA/NM, } \\
\text { NA/NM }\end{array}$ & , Yes & $\begin{array}{l}\text { Medulla } \\
\text { oblongata }\end{array}$ & OA, AphA & Anterior spinal vein & Type V & TAE & Completely & 2 months & No & 3 \\
\hline $\begin{array}{l}80 \text { Copelan et al. } \\
\text { (66) }\end{array}$ & $59 / \mathrm{M}$ & $\begin{array}{l}\text { Dizziness, nausea, } \\
\text { and vomiting, } \\
\text { vertigo/5 weeks }\end{array}$ & No & SPS & No & $\begin{array}{l}\text { NANM, mild } \\
\text { patchy } \\
\text { enhancement }\end{array}$ & Hyper & Hyper & $\begin{array}{l}\text { Mild } \\
\text { hyper, } \\
\text { hyper }\end{array}$ & Yes & $\begin{array}{l}\text { Medulla } \\
\text { oblongata } \\
\text { extending to the } \\
\text { upper cervical } \\
\text { cord }\end{array}$ & $\begin{array}{l}\text { MMA, OA, } \\
\text { AphA }\end{array}$ & $\begin{array}{l}\text { Petrosal } \\
\text { vein } \rightarrow \text { PMV }\end{array}$ & Type V & $\begin{array}{l}\text { Combination of } \\
\text { endovascular } \\
\text { embilization and } \\
\text { surgical resection }\end{array}$ & Completely & 3 years & No & 1 or 2 \\
\hline 81 & $72 / \mathrm{M}$ & $\begin{array}{l}\text { Slurred speech, } \\
\text { and dysphagia/3 } \\
\text { months }\end{array}$ & No & $\begin{array}{l}\text { CGJ } \\
\text { (anterior } \\
\text { condylar } \\
\text { vein) }\end{array}$ & No & $\begin{array}{l}\text { NA/NM, } \\
\text { enhanced }\end{array}$ & Hyper & NAINM & $\begin{array}{l}\text { NA/NM, } \\
\text { NAINM }\end{array}$ & , Yes & $\begin{array}{l}\text { Medulla } \\
\text { oblongata and } \\
\text { cerebellar } \\
\text { fllocculus }\end{array}$ & AphA & $\begin{array}{l}\text { Anterior condylar } \\
\text { vein, petrosal vein } \\
\text { and PMV }\end{array}$ & Type V & $\begin{array}{l}\text { TAE with Onyx via } \\
\text { AphA }\end{array}$ & Completely & 5 months & No & 1 or 2 \\
\hline 82 & $35 / F$ & $\begin{array}{l}\text { Progressive } \\
\text { unsteady gait and } \\
\text { paraparesis/1 } \\
\text { month }\end{array}$ & No & SPS & No & $\begin{array}{l}\text { NA/NM, mildly } \\
\text { enhanced }\end{array}$ & Hyper & NANM & $\begin{array}{l}\text { NA/NM, } \\
\text { NA/NM }\end{array}$ & , Yes & $\begin{array}{l}\text { Medulla } \\
\text { oblongata }\end{array}$ & $O A$ & $\mathrm{SPS} \rightarrow \mathrm{PMV}$ & Type V & TAE with Onyx & Completely & 3months & No & 2 \\
\hline 83 & $64 / \mathrm{M}$ & $\begin{array}{l}\text { Tetraparesis/6 } \\
\text { months }\end{array}$ & $\begin{array}{l}\text { Yes (transverse } \\
\text { myelitis) }\end{array}$ & SPS & No & $\begin{array}{l}\text { NA/NM, } \\
\text { enhanced }\end{array}$ & Hyper & NAINM & $\begin{array}{l}\text { NA/NM, } \\
\text { NAINM }\end{array}$ & Yes & $\begin{array}{l}\text { Medulla } \\
\text { oblongata } \\
\text { extending to the } \\
\text { upper cervical } \\
\text { cord }\end{array}$ & $\begin{array}{l}\text { MHT of ICA, } \\
\text { OA }\end{array}$ & PMV & Type V & $\begin{array}{l}\text { TAE and surgical } \\
\text { resection }\end{array}$ & Completely & 12 months & No & 4 or 5 \\
\hline $\begin{array}{l}84 \text { Rodriguez et al. } \\
\text { (67) }\end{array}$ & $68 / \mathrm{M}$ & $\begin{array}{l}\text { Progressivive hypoer } \\
\text { extremity } \\
\text { weakness/NANM }\end{array}$ & & Tentorium & No & $\begin{array}{l}\text { NA/NM, } \\
\text { NA/NM }\end{array}$ & NAINM & NAINM & $\begin{array}{l}\text { NA/NM, } \\
\text { NAINM }\end{array}$ & & $\begin{array}{l}\text { Cenvicomedullary } \\
\text { junction to } \mathrm{C7}\end{array}$ & PMA & $\begin{array}{l}\text { Doral and ventral } \\
\text { perimedullary veins }\end{array}$ & Type V & $\begin{array}{l}\text { TAE with 50\% } \\
\text { ethanol }\end{array}$ & Incompletely & NANM & $\begin{array}{l}\text { Yes/open } \\
\text { surgery }\end{array}$ & 3 \\
\hline
\end{tabular}




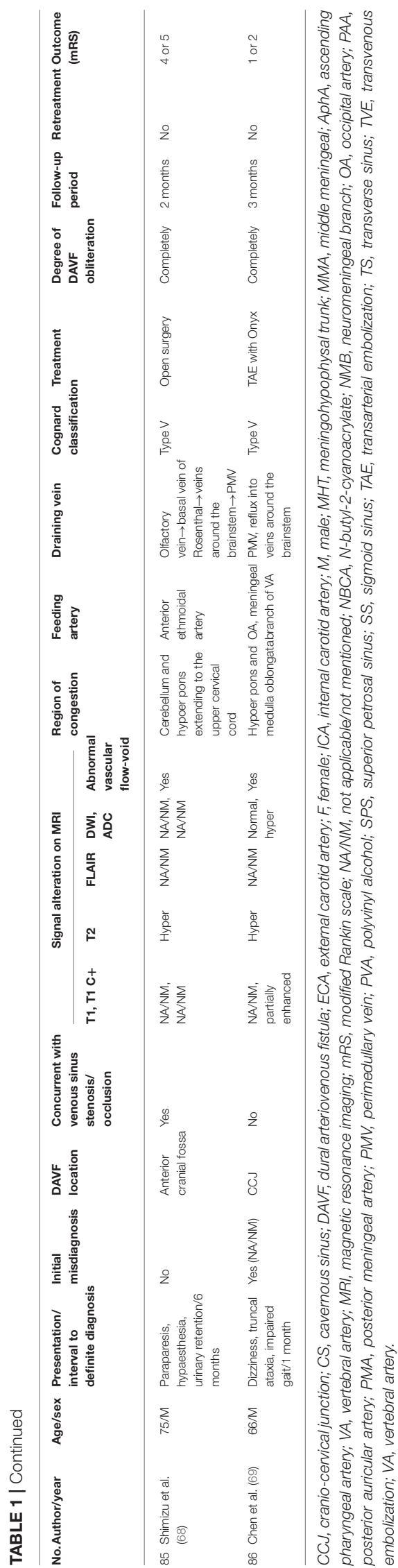

draining system might play an important role in the genesis of brainstem engorgement in patients with intracranial DAVFs.

The brainstem has a complex venous draining system. In general, the veins of the brainstem can be divided into the transverse and longitudinal groups, which are named on the basis of the subdivision (mesencephalon, pons, or medulla), surface (median anterior, lateral anterior, or lateral), and the direction (transverse or longitudinal) of the brainstem drained (71). From cranial to caudal, the transverse groups are peduncular vein, posterior communicating vein, vein of pontomesencephalic sulcus, transverse pontine vein, vein of pontomedullary sulcus, and transverse medullary vein. From median to lateral, the longitudinal groups are median veins (median anterior pontomesencephalic vein, median anterior medullary vein), anterolateral veins (lateral anterior pontomesencephalic vein, lateral anterior medullary vein), and lateral veins (lateral mesencephalic vein, lateral medullary and retro-olivary veins). The veins of the transverse group have extensive anastomoses with those of the longitudinal group. Besides, the terminal end of the veins draining the brainstem and cerebellum form bridging veins that are divided into three groups: (1) a galenic group draining into the vein of Galen; (2) a petrosal group draining into the petrosal sinuses; and (3) a tentorial group draining into the sinuses converging on the torcula. Hence, DAVFs in the posterior fossa or even cavernous sinus could lead to brainstem engorgement. Venous drainage of the brainstem is presented in Figure 5.

The diagnosis of intracranial DAVFs with brainstem engorgement is still challenging. Patients that were diagnosed with neoplasm to undergo brainstem biopsy or given corticosteroids for misdiagnosing as myelitis were not uncommonly reported $(4,51)$. According to our analysis, $40.2 \%(33 / 82)$ of the patients were initially misdiagnosed as other diseases. Of note, the rate of initial misdiagnosis did not decrease in the past three decades (Figure 6). Considering the unspecific clinical manifestations of intracranial DAVFs with brainstem engorgement, meticulous and comprehensive interpretation of the auxiliary investigations is of utmost importance.

While conventional angiography is the gold standard for definite diagnosis of intracranial DAVFs, taking good advantage of different sequences of MRI data could help screen out those patients with high suspicion. Abnormal vascular flow voids on MRI are reliable evidence highly suggestive of vascular lesions. Abnormal vascular flow voids could only be identified in $80.2 \%$ $(69 / 86)$ of the patients in this survey, including those identified after repeated review of the MRI or those identified during multiple investigations of MRI after symptom aggravation. T2WI or FLAIR sequence is highly sensitive (in 100\% of the patients) for the engorged brainstem but with low specificity. The signals on T1WI are so polytropic that $65.2 \%(15 / 23)$ of the analyzed patients presented with low hypointensity and $34.8 \%(8 / 23)$ of the patients were normal. The engorged brainstem was enhanced on T1WI with different degrees in $72.5 \%$ (37/51) of the patients after gadolinium contrast. DWI and ADC were rarely performed in these patients. All of the six patients with ADC map showed hyperintensity which denotes the vascular origin of brainstem edema. The signal of DWI is so variable that heterogeneous, 


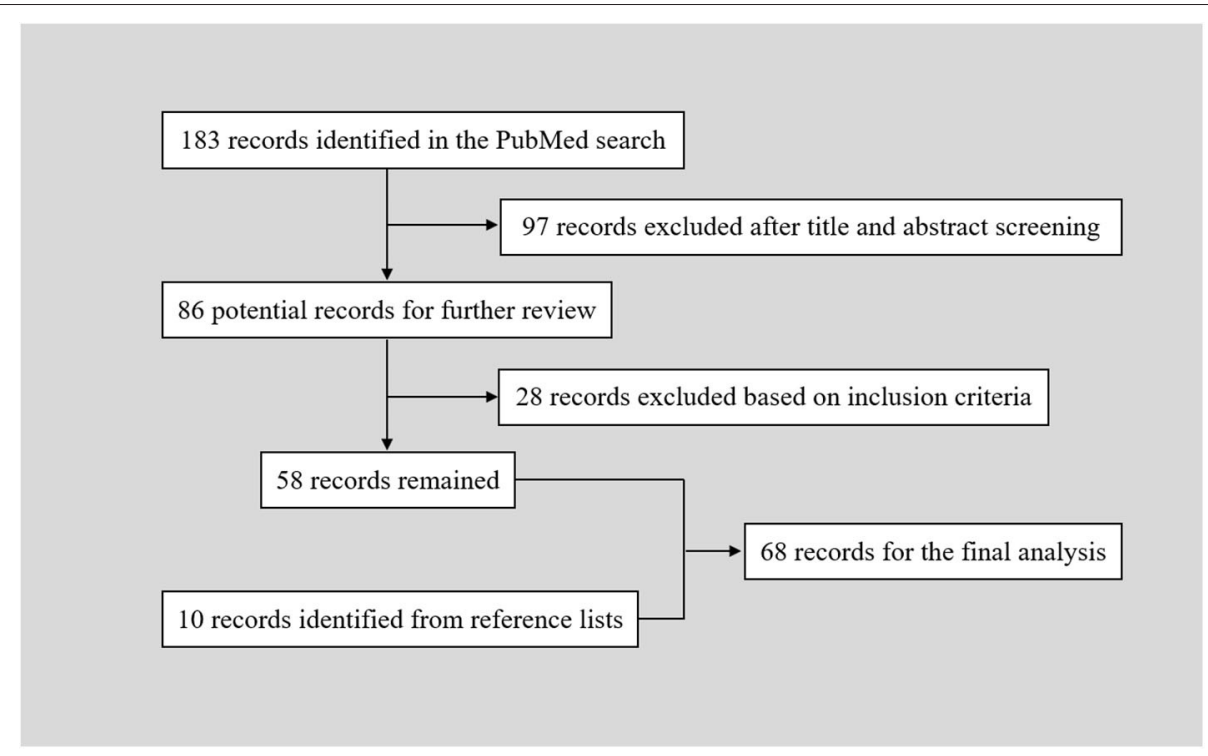

FIGURE 2 | Flow chart of the searching strategy.

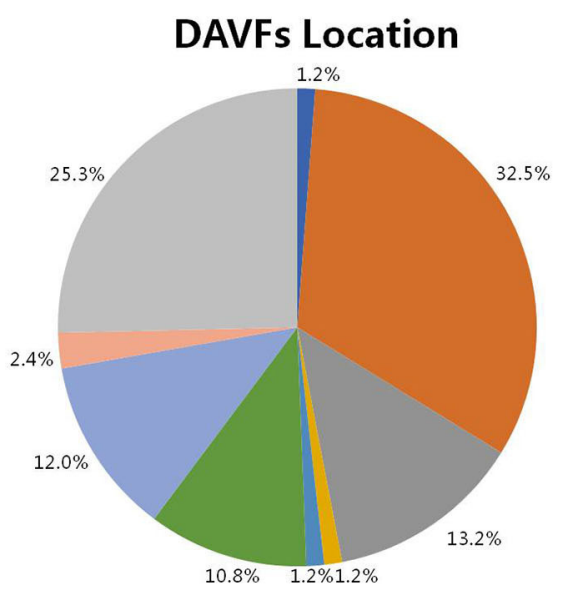

- anterior fossa

n cranio-cervical junction

a cavernous sinus

- vein of Galen

- occipital sinus

n superior petrosal sinus

- transverse/sigmoid sinus

torcular

tentorium

FIGURE 3 | The specific location of intracranial DAVFs complicated with brainstem engorgement. DAVF, dural arteriovenous fistula.

hyper, hypo, and normal intensity could be in 1 (10\%), 3 (30\%), $1(10 \%)$, and $5(50 \%)$ of the 10 identified patients, which might reflect the different degree and duration of venous congestion around the brainstem. Furthermore, contrast-enhanced dynamic magnetic resonance angiography is more sensitive to find out occult vascular abnormalities $(50,72)$. T2*WI and susceptibilityweighted imaging are emerging sequences of MRI that are good at detecting fine vasculature and microbleeds (73). Hypointense signal could be noticed in the engorged brainstem on $\mathrm{T} 2{ }^{*} \mathrm{WI}$ and susceptibility-weighted imaging, for long-term venous congestion might lead to intraparenchymal microbleeding in the brainstem $(57,60)$. Besides, some authors also demonstrated decreased cerebral blood volume and prolongation of the mean transit time on magnetic resonance perfusion in the engorged brainstem (66). Hence, advanced MRI sequences could increase the sensitivity and specificity in differential diagnosis of lesion nature and avoid delayed treatment and unnecessary conventional angiography.

There is no consensus on the treatment option for intracranial DAVFs with brainstem engorgement. Of note, premature administration of corticosteroid could be dangerous even fatal in case of undiagnosed DAVFs with brainstem or spinal cord engorgement $(74,75)$. Hence, precise and comprehensive diagnosis is crucial for further treatment. The treatment should be based on the specific angioarchitecture, intracranial location, and technique availability. Generally speaking, the treatment strategies for DAVFs include open surgery, endovascular embolization, and radiotherapy. As the lag time of effect could be up to 3 years (76), radiotherapy is unsuitable for patients with brainstem engorgement. With the development of endovascular 


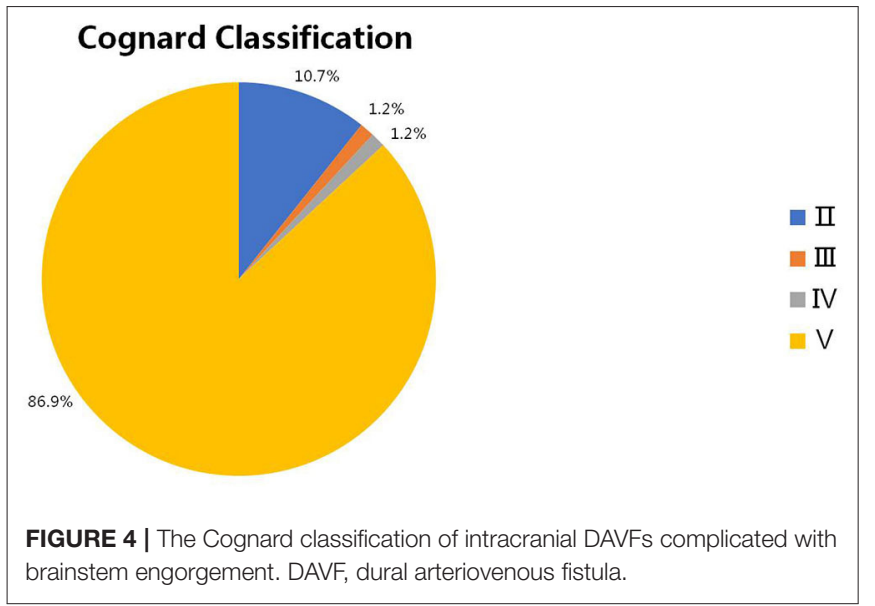

technique and materials, endovascular embolization has become the first-line choice for the majority of intracranial DAVFs (63, 70). Besides, endovascular treatment can be an adjunctive step of further open surgery. For patients with difficult arterial/venous access, incomplete fistula obliteration, recanalization after embolization, open surgery can be considered. Whereas, in patients where a transfemoral approach is impaired for the tortuosity of feeding arteries or the presence of isolated sinuses, percutaneous or intraoperative puncture of perforating arteries or draining veins and venous sinuses represent a new choice to facilitate distal access to the DAVFs $(70,77)$. In this review, $63.1 \%$ of the patients were treated endovascularly (transarterial or transvenous), $26.2 \%$ of the patients underwent open surgery, and $9.5 \%$ of the patients were treated conjointly with endovascular and open surgical approaches.
A

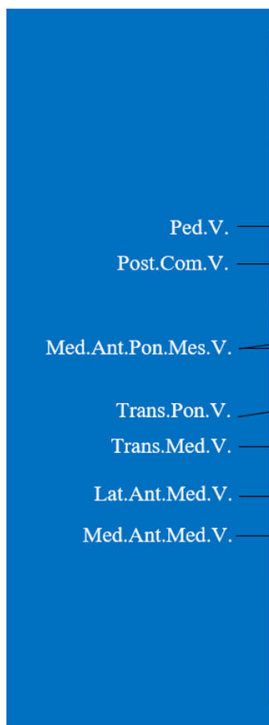

B

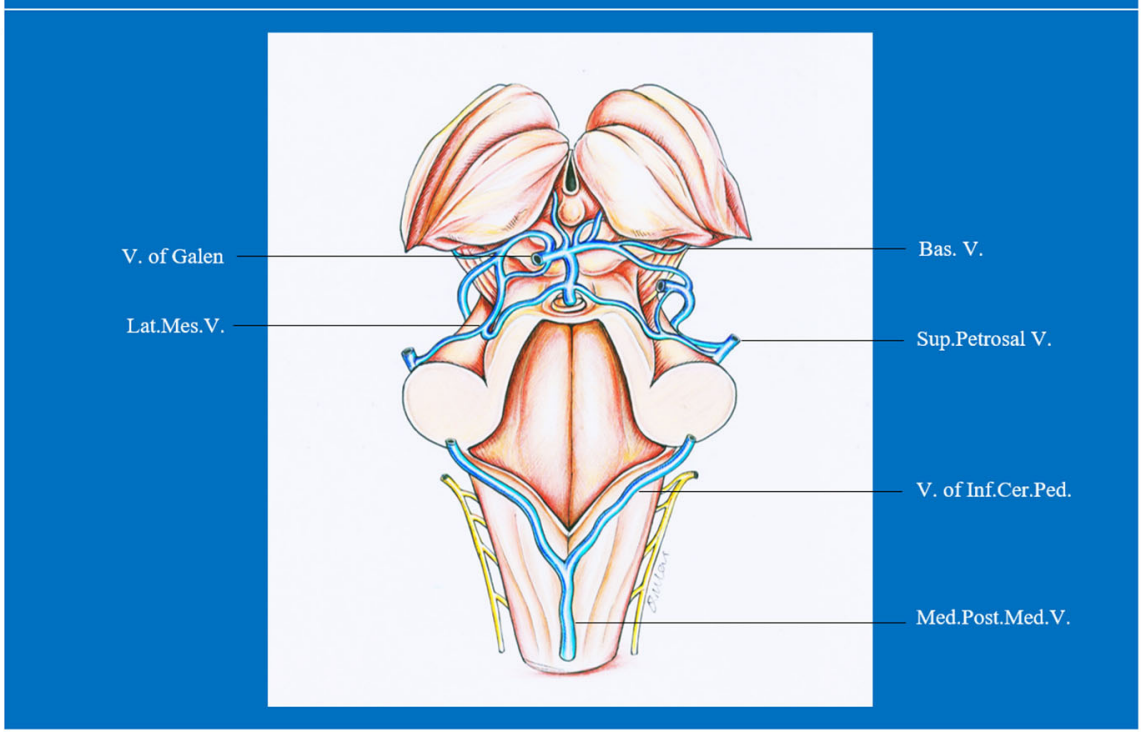

FIGURE 5 | Ventral (A) and dorsal (B) venous drainage of the brainstem. Ant., anterior; Bas., basilar; Br., bridging; Cer., cerebellar; Com., communicating; Inf., inferior; Lat., lateral; Med., median, medullary; Mes., mesencephalic; Ped., peduncle; Pon., pontine; Post., posterior; Sul., sulcus; Sup., superior; Trans., transverse; Trig., trigeminal; V., vein. 


\section{The state of diagnosis and treatment of intracranial DAVFs with \\ brainstem engorgement in the past 3 decades}

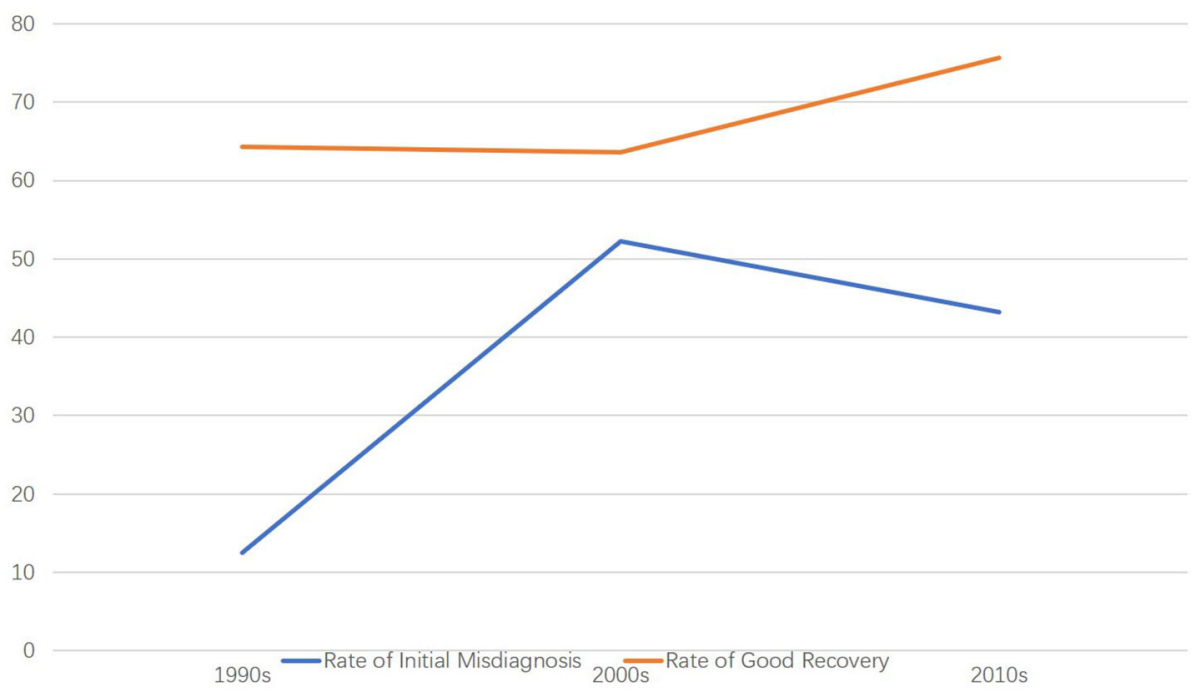

FIGURE 6 | The state of diagnosis and treatment of intracranial DAVFs complicated with brainstem engorgement in the past three decades.

The prognosis of patients with DAVFs associated brainstem engorgement is still unsatisfactory, though slight increase in good recovery could be noted in the past three decades (Figure 3). Only $70 \%$ of the patients experienced a good recovery (mRS score $\leq 3$ ). A substantial number of patients can have more or less neurological deficits. Except for the peculiar location of DAVFs, angioarchitecture, and surrounding neural structures, early diagnosis is the most important factor impacting prognosis. According to this review, correct diagnosis could be achieved in only $50 \%$ of the patients in the first 3 months after symptom onset. What's more, the rate of initial misdiagnosis did not decrease in the past three decades (Figure 3). Hence, early awareness of this rare entity and efficiently utilizing the up to date investigations are of utmost importance.

\section{LIMITATIONS}

The opinion of this review was deduced from retrospective review of the published case reports or small case series. The results would be biased by many factors. Firstly, the levels in diagnosis and treatment vary greatly between different centers. Secondly, due to the reporting customs among different authors, a lot of key information was missing. Thirdly, the mean followup period was only 7.86 ( $n=74$, range $0-60$ months) months,

\section{REFERENCES}

1. Kuwayama N. Epidemiologic survey of dural arteriovenous fistulas in japan: clinical frequency and present status of treatment. Acta Neurochir Suppl. (2016) 123:185-8. doi: 10.1007/978-3-319-298 87-0_26 which could impair the accuracy in outcome assessment. Of note, there were two studies reporting larger case series of DAVFs with brainstem engorgement $(63,77)$ that were not included in this analysis because so much information was missing according to our inclusion criteria.

\section{DATA AVAILABILITY STATEMENT}

The datasets generated for this study are available on request to the corresponding author.

\section{AUTHOR CONTRIBUTIONS}

JY contributed to the conception and design of the manuscript. LQ and HL performed literature review. KH and GL wrote the manuscript. KX and JY critically revised the manuscript. All authors contributed to the article and approved the submitted version.

\section{FUNDING}

This research received funding support from the Ninth Youth Scientific Research Funding of The First Hospital of Jilin University (jdyy92018035). 
4. Zhang S, Liu H, Li J. Cervical myelopathy caused by intracranial dural arteriovenous fistula with acute worsening after steroid administration. World Neurosurg. (2018) 120:328-30. doi: 10.1016/j.wneu.2018.09.029

5. Probst EN, Christante L, Zeumer H. Brain-stem venous congestion due to a dural arteriovenous fistula in the posterior fossa. J Neurol. (1994) 241:175-7. doi: 10.1007/BF00868346

6. Uchino A, Kato A, Kuroda Y, Shimokawa S, Kudo S. Pontine venous congestion caused by dural carotid-cavernous fistula: report of two cases. Eur Radiol. (1997) 7:405-8. doi: 10.1007/s003300050175

7. Ernst RJ, Gaskill-Shipley M, Tomsick TA, Hall LC, Tew JM Jr, et al. Cervical myelopathy associated with intracranial dural arteriovenous fistula: MR findings before and after treatment. AJNR Am J Neuroradiol. (1997) $18: 1330-4$.

8. Ricolfi F, Manelfe C, Meder JF, Arrue P, Decq P, Brugieres P, et al. Intracranial dural arteriovenous fistulae with perimedullary venous drainage. Anatomical, clinical and therapeutic considerations. Neuroradiology. (1999) 41:803-12. doi: $10.1007 / \mathrm{s} 002340050846$

9. Bousson V, Brunereau L, Vahedi K, Chapot R. Intracranial dural fistula as a cause of diffuse MR enhancement of the cervical spinal cord. J Neurol Neurosurg Psychiatry. (1999) 67:227-30. doi: 10.1136/jnnp.6 7.2.227

10. Hurst RW, Bagley LJ, Scanlon M, Flamm ES. Dural arteriovenous fistulas of the craniocervical junction. Skull Base Surg. (1999) 9:1-7. doi: 10.1055/s-2008-1058166

11. Takahashi S, Tomura N, Watarai J, Mizoi K, Manabe H. Dural arteriovenous fistula of the cavernous sinus with venous congestion of the brain stem: report of two cases. AJNR Am J Neuroradiol. (1999) 20:886-8.

12. Shintani S, Tsuruoka S, Shiigai T. Carotid-cavernous fistula with brainstem congestion mimicking tumor on MRI. Neurology. (2000) 55:1929-31. doi: 10.1212/WNL.55.12.1929

13. Wiesmann M, Padovan CS, Pfister HW, Yousry TA. Intracranial dural arteriovenous fistula with spinal medullary venous drainage. Eur Radiol. (2000) 10:1606-9. doi: 10.1007/s003300000382

14. Kalamangalam GP, Bhattacharya J, Teasdale E, Thomas M. Myelopathy from intracranial dural arteriovenous fistula. J Neurol Neurosurg Psychiatry. (2002) 72:816-8. doi: 10.1136/jnnp.72.6.816

15. Weigele JB, Chaloupka JC, Lesley WS. Galenic dural arteriovenous fistula: unusual clinical presentation and successful endovascular therapy. Case report. J Neurosurg. (2002) 97:467-70. doi: 10.3171/jns.2002.97. 2.0467

16. Asakawa H, Yanaka K, Fujita K, Marushima A, Anno I, Nose T. Intracranial dural arteriovenous fistula showing diffuse MR enhancement of the spinal cord: case report and review of the literature. Surg Neurol. (2002) 58:251-7. doi: 10.1016/S0090-3019(02)00861-3

17. Lanz M, Thiemann U, Grzyska U, Ebke M, Schwendemann G, Kraus JA. Transient brainstem ischemia and recurrent syncope caused by a dural arteriovenous fistula. Neurology. (2003) 61:1152-3. doi: 10.1212/WNL.61.8.1152

18. Kai Y, Hamada JI, Morioka M, Yano S, Ushio Y. Brain stem venous congestion due to dural arteriovenous fistulas of the cavernous sinus. Acta Neurochir. (2004) 146:1107-11. doi: 10.1007/s00701-004-0315-3

19. Li J, Ezura M, Takahashi A, Yoshimoto T. Intracranial dural arteriovenous fistula with venous reflux to the brainstem and spinal cord mimicking brainstem infarction-case report. Neurol Med Chir. (2004) 44:24-8. doi: 10.2176/nmc.44.24

20. Pannu Y, Shownkeen H, Nockels RP, Origitano TC. Obliteration of a tentorial dural arteriovenous fistula causing spinal cord myelopathy using the cranio-orbito zygomatic approach. Surg Neurol. (2004) 62:463-7. doi: 10.1016/j.surneu.2004.01.017

21. Crum BA, Link M. Intracranial dural arteriovenous fistula mimicking brainstem neoplasm. Neurology. (2004) 62:2330-1. doi: 10.1212/01.WNL.0000130342.68494.78

22. Oishi H, Horinaka N, Shmizu T, Ozaki Y, Arai H. A case of intracranial dural arteriovenous fistula presenting with brainstem infarction. No Shinkei Geka. (2005) 33:1095-9. doi: 10.11477/mf.1436100147

23. Satoh M, Kuriyama M, Fujiwara T, Tokunaga K, Sugiu K. Brain stem ischemia from intracranial dural arteriovenous fistula: case report. Surg Neurol. (2005) 64:341-5. doi: 10.1016/j.surneu.2004.12.029
24. Akkoc Y, Atamaz F, Oran I, Durmaz B. Intracranial dural arteriovenous fistula draining into spinal perimedullary veins: a rare cause of myelopathy. J Korean Med Sci. (2006) 21:958-62. doi: 10.3346/jkms.2006.21.5.958

25. Iwasaki M, Murakami K, Tomita T, Numagami Y, Nishijima M. Cavernous sinus dural arteriovenous fistula complicated by pontine venous congestion. A case report. Surg Neurol. (2006) 65:516-8. doi: 10.1016/j.surneu.2005.06.044

26. Lagares A, Perez-Nunez A, Alday R, Ramos A, Campollo J, Lobato RD. Dural arteriovenous fistula presenting as brainstem ischaemia. Acta Neurochir. (2007) 149:965-7. doi: 10.1007/s00701-007-1250-x

27. van Rooij WJ, Sluzewski M, Beute GN. Intracranial dural fistulas with exclusive perimedullary drainage: the need for complete cerebral angiography for diagnosis and treatment planning. AJNR Am J Neuroradiol. (2007) 28:34851. doi: 10.1016/S0098-1672(08)70215-7

28. Sakamoto S, Ohba S, Shibukawa M, Kiura Y, Okazaki T, Kurisu K. Course of apparent diffusion coefficient values in cerebral edema of dural arteriovenous fistula before and after treatment. Clin Neurol Neurosurg. (2008) 110:400-3. doi: 10.1016/j.clineuro.2007.12.010

29. Tsutsumi S, Yasumoto Y, Ito M, Oishi H, Arai H. Posterior fossa dural arteriovenous fistula as a probable cause of congestive myelopathy. Case report. Neurol Med Chir. (2008) 48:171-5. doi: 10.2176/nmc.48.171

30. Sugiura Y, Nozaki T, Sato H, Sawashita K, Hiramatsu H, Nishizawa S. Sigmoid sinus dural arteriovenous fistula with spinal venous drainage manifesting as only brainstem-related neurological deficits without myelopathy: case report. Neurol Med Chir. (2009) 49:71-6. doi: 10.2176/nmc.49.71

31. Wang HC, Lin WC, Kuo YL, Yang TM, Ho JT, Tsai NW, et al. Factors associated with brainstem congestive encephalopathy in dural arterio-venous fistulas. Clin Neurol Neurosurg. (2009) 111:335-40. doi: 10.1016/j.clineuro.2008.11.004

32. Khan S, Polston DW, Shields RW, Jr, Rasmussen P, Gupta R. Tentorial dural arteriovenous fistula presenting with quadriparesis: case report and review of the literature. J Stroke Cerebrovasc Dis. (2009) 18:428-34. doi: 10.1016/j.jstrokecerebrovasdis.2008.12.007

33. Ko SB, Kim CK, Lee SH, Yoon BW. Carotid cavernous fistula with cervical myelopathy. J Clin Neurosci. (2009) 16:1350-3. doi: 10.1016/j.jocn.2008.12.031

34. Kleeberg J, Maeder-Ingvar M, Maeder P. Progressive cervical myelopathy due to dural craniocervical fistula. Eur Neurol. (2010) 63:374. doi: $10.1159 / 000292430$

35. Patsalides A, Tzatha E, Stubgen JP, Shungu DC, Stieg PE, Gobin YP. Intracranial dural arteriovenous fistula presenting as an enhancing lesion of the medulla. J Neurointerv Surg. (2010) 2:390-3. doi: 10.1136/jnis.2009.001750

36. Aixut Lorenzo S, Tomasello Weitz A, Blasco Andaluz J, Sanroman Manzanera L, Macho Fernandez JM. Transvenous approach to intracranial dural arteriovenous fistula (Cognard v): a treatment option. A case report. Interv Neuroradiol. (2011) 17:108-14. doi: 10.1177/159101991101700117

37. Kim NH, Cho KT, Seo HS. Myelopathy due to intracranial dural arteriovenous fistula: a potential diagnostic pitfall. Case report. J Neurosurg. (2011) 114:8303. doi: 10.3171/2010.5.JNS10128

38. Peltier J, Baroncini M, Thines L, Lacour A, Leclerc X, Lejeune JP. Subacute involvement of the medulla oblongata and occipital neuralgia revealing an intracranial dural arteriovenous fistula of the craniocervical junction. Neurol India. (2011) 59:285-8. doi: 10.4103/0028-3886.79153

39. Clark SW, Dang T, Toth G, Pride GL, Greenberg B, Warnack W. Carotid cavernous fistula imitating brainstem glioma. Arch Neurol. (2011) 68:256-7. doi: 10.1001/archneurol.2010.366

40. Ogbonnaya ES, Yousaf I, Sattar TM. Intracranial dural arterio-venous fistula presenting with progressive myelopathy. BMJ Case Rep. (2011) 2011:4828. doi: 10.1136/bcr.09.2011.4828

41. Kulwin C, Bohnstedt BN, Scott JA, Cohen-Gadol A. Dural arteriovenous fistulas presenting with brainstem dysfunction: diagnosis and surgical treatment. Neurosurg Focus. (2012) 32:E10. doi: 10.3171/2012.2.FOCUS1217

42. Clark CN, Saifee TA, Cowley PO, Ginsberg L. Dural arteriovenous fistula of the medulla initially mimicking Guillain-Barre syndrome. Arch Neurol. (2012) 69:786-7. doi: 10.1001/archneurol.2011.2934

43. Mathon B, Gallas S, Tuillier T, Bekaert O, Decq P, Brugieres P, et al. Intracranial dural arteriovenous fistula with perimedullary venous drainage: anatomical, clinical and therapeutic considerations about one case, and review of the literature. Neurochirurgie. (2013) 59:133-7. doi: 10.1016/j.neuchi.2013.04.009 
44. Salamon E, Patsalides A, Gobin YP, Santillan A, Fink ME. Dural arteriovenous fistula at the craniocervical junction mimicking acute brainstem and spinal cord infarction. JAMA Neurol. (2013) 70:796-7. doi: 10.1001/jamaneurol.2013.1946

45. Singh D, Garg A, Gupta A, Goel G, Gupta R, Bansal A. Tentorial dural arteriovenous fistula presenting as episodic weakness mimicking periodic paralysis. J Neurointerv Surg. (2013) 5:e32. doi: 10.1136/neurintsurg-2012-010281

46. El Asri AC, El Mostarchid B, Akhaddar A, Naama O, Gazzaz M, Boucetta M. Factors influencing the prognosis in intracranial dural arteriovenous fistulas with perimedullary drainage. World Neurosurg. (2013) 79:182-91. doi: 10.1016/j.wneu.2012.09.012

47. Foreman SM, Stahl MJ, Schultz GD. Paraplegia in a chiropractic patient secondary to atraumatic dural arteriovenous fistula with perimedullary hypertension: case report. Chiropr Man Therap. (2013) 21:23. doi: 10.1186/2045-709X-21-23

48. Gross R, Ali R, Kole M, Dorbeistein C, Jayaraman MV, Khan M. Tentorial dural arteriovenous fistula presenting as myelopathy: case series and review of literature. World J Clin Cases. (2014) 2:907-11. doi: 10.12998/wjcc.v2.i12.907

49. Wu Q, Wang HD, Shin YS, Zhang X. Brainstem congestion due to dural ateriovenous fistula at the craniocervical junction. J Korean Neurosurg Soc. (2014) 55:152-5. doi: 10.3340/jkns.2014.55.3.152

50. Haryu S, Endo T, Sato K, Inoue T, Takahashi A, Tominaga T. Cognard type V intracranial dural arteriovenous shunt: case reports and literature review with special consideration of the pattern of spinal venous drainage. Neurosurgery. (2014) 74:E135-42. doi: 10.1227/NEU.0000000000000069

51. Roelz R, Van Velthoven V, Reinacher P, Coenen VA, Mader I, Urbach $\mathrm{H}$, et al. Unilateral contrast-enhancing pontomedullary lesion due to an intracranial dural arteriovenous fistula with perimedullary spinal venous drainage: the exception that proves the rule. J Neurosurg. (2015) 123:1534-9. doi: $10.3171 / 2014.11$.JNS142278

52. Le Guennec L, Leclercq D, Szatmary Z, Idbaih A, Reyes-Botero G, Delattre JY, et al. Dural arteriovenous fistula mimicking a brainstem glioma. $J$ Neuroimaging. (2015) 25:1053-5. doi: 10.1111/jon.12220

53. Alvarez H, Sasaki-Adams D, Castillo M. Resolution of brainstem edema after treatment of a dural tentorial arteriovenous fistula. Interv Neuroradiol. (2015) 21:603-8. doi: 10.1177/1591019915591741

54. Pop R, Manisor M, Aloraini Z, Chibarro S, Proust F, Quenardelle V, et al. Foramen magnum dural arteriovenous fistula presenting with epilepsy. Interv Neuroradiol. (2015) 21:724-7. doi: 10.1177/1591019915609783

55. Abud LG, Abud TG, Nakiri GS, Queiroz RM, Abud DG. Intracranial dural arteriovenous fistula with perimedullary drainage treated by endovascular embolization. Arq Neuropsiquiatr. (2016) 74:178-9. doi: 10.1590/0004-282X20150171

56. Abdelsadg M, Kanodia AK, Keston P, Galea J. Unusual case of intracranial dural AV fistula presenting with acute myelopathy. BMJ Case Rep. (2016) 2016:bcr2016215227. doi: 10.1136/bcr-2016-215227

57. Enokizono M, Sato N, Morikawa M, Kimura Y, Sugiyama A, Maekawa T, et al. "Black butterfly" sign on T2*-weighted and susceptibility-weighted imaging: A novel finding of chronic venous congestion of the brain stem and spinal cord associated with dural arteriovenous fistulas. J Neurol Sci. (2017) 379:64-8. doi: 10.1016/j.jns.2017.05.066

58. Tanaka J, Fujita A, Maeyama M, Kohta M, Hosoda K, Kohmura E. Cognard type Vdural arteriovenous fistula involving the occipital sinus. J Stroke Cerebrovasc Dis. (2017) 26:e62-3. doi: 10.1016/j.jstrokecerebrovasdis.2017.01.004

59. Emmer BJ, van Es AC, Koudstaal PJ, Roosendaal SD. Infratentorial dural arteriovenous fistula resulting in brainstem edema and enhancement. Neurology. (2017) 88:503-4. doi: 10.1212/WNL.0000000000003569

60. Duan SS, Liu H, Wang WL, Zhao CB. A case of intracranial dural arteriovenous fistula mimicking brainstem tumor. Chin Med J. (2017) 130:2519-20. doi: 10.4103/0366-6999.216398

61. Chen PM, Chen MM, McDonald M, McGehrin K, Steinberg J, Handwerker J, et al. Cranial Dural Arteriovenous Fistula. Stroke. (2018) 49:e332-4. doi: 10.1161/STROKEAHA.118.022508

62. Bernard F, Lemee JM, Faguer R, Fournier HD. Lessons to be remembered from a dural arteriovenous fistula mimicking medulla and high cervical cord glioma. World Neurosurg. (2018) 113:312-5. doi: 10.1016/j.wneu.2018 02.161

63. Li J, Ren J, Du S, Ling F, Li G, Zhang H. Dural arteriovenous fistulas at the petrous apex. World Neurosurg. (2018) 119:e968-e76. doi: 10.1016/j.wneu.2018.08.012

64. Wang XC, Du YY, Tan Y, Qin JB, Wang L, Wu XF, et al. Brainstem congestion due to dural arteriovenous fistula at the craniocervical junction: case report and review of the literature. World Neurosurg. (2018) 118:181-7. doi: 10.1016/j.wneu.2018.06.243

65. Takahashi H, Ueshima T, Goto D, Kimura T, Yuki N, Inoue Y, et al. acute tetraparesis with respiratory failure after steroid administration in a patient with a dural arteriovenous fistula at the craniocervical junction. Intern Med. (2018) 57:591-4. doi: 10.2169/internalmedicine.9115-17

66. Copelan AZ, Krishnan A, Marin H, Silbergleit R. Dural arteriovenous fistulas: a characteristic pattern of edema and enhancement of the medulla on MRI. AJNR Am J Neuroradiol. (2018) 39:238-44. doi: 10.3174/ajnr.A5460

67. Rodriguez Rubio R, Chae R, Rutledge WC, De Vilalta A, Kournoutas I, Winkler E, et al. Clipping of a high-risk dural arteriovenous fistula of the posterior fossa: 3d operative video. World Neurosurg. (2019) 126:413. doi: 10.1016/j.wneu.2019.03.101

68. Shimizu A, Ishikawa T, Yamaguchi K, Funatsu T, Ryu B, Nagahara A, et al. Brainstem venous congestion caused by perimedullary drainage in anterior cranial fossa dural arteriovenous fistula. World Neurosurg. (2019) 127:503-8. doi: 10.1016/j.wneu.2019.04.204

69. Chen PY, Juan YH, Lin SK. An isolated unilateral pontomedullary lesion due to an intracranial dural arteriovenous fistula mimicking a brain tumor - case and review. J Nippon Med Sch. (2019) 86:48-54. doi: 10.1272/jnms.JNMS.2019_86-9

70. Reynolds MR, Lanzino G, Zipfel GJ. Intracranial dural arteriovenous fistulae. Stroke. (2017) 48:1424-31. doi: 10.1161/STROKEAHA.116.012784

71. Rhoton AL Jr. The posterior fossa veins. Neurosurgery. (2000) 47(Suppl.3):S69-92. doi: 10.1093/neurosurgery/47.3.S69

72. Meckel S, Maier M, Ruiz DS, Yilmaz H, Scheffler K, Radue EW, et al. MR angiography of dural arteriovenous fistulas: diagnosis and follow-up after treatment using a time-resolved 3D contrast-enhanced technique. AJNR Am J Neuroradiol. (2007) 28:877-84.

73. Di Ieva A, Lam T, Alcaide-Leon P, Bharatha A, Montanera W, Cusimano MD. Magnetic resonance susceptibility weighted imaging in neurosurgery: current applications and future perspectives. J Neurosurg. (2015) 123:1463-75. doi: 10.3171/2015.1.JNS142349

74. Zalewski NL, Rabinstein AA, Brinjikji W, Kaufmann TJ, Nasr D, Ruff MW, et al. Unique gadolinium enhancement pattern in spinal dural arteriovenous fistulas. JAMA Neurol. (2018) 75:1542-5. doi: 10.1001/jamaneurol.20 18.2605

75. Nasr DM, Brinjikji W, Rabinstein AA, Lanzino G. Clinical outcomes following corticosteroid administration in patients with delayed diagnosis of spinal arteriovenous fistulas. J Neurointerv Surg. (2017) 9:607-10. doi: 10.1136/neurintsurg-2016-012430

76. Soderman M, Edner G, Ericson K, Karlsson B, Rahn T, Ulfarsson E, et al. Gamma knife surgery for dural arteriovenous shunts: 25 years of experience. J Neurosurg. (2006) 104:867-75. doi: 10.3171/jns.2006.104.6.867

77. Luo CB, Chang FC, Teng MM, Lin CJ, Wang AG, Ting TW. Aggressive cavernous sinus dural arteriovenous fistula: angioarchitecture analysis and embolization by various approaches. J Chin Med Assoc. (2016) 79:152-8. doi: 10.1016/j.jcma.2015.09.001

Conflict of Interest: The authors declare that the research was conducted in the absence of any commercial or financial relationships that could be construed as a potential conflict of interest.

Copyright (c) $2020 \mathrm{Hou}, \mathrm{Li}, \mathrm{Qu}, \mathrm{Liu}, \mathrm{Xu}$ and $\mathrm{Yu}$. This is an open-access article distributed under the terms of the Creative Commons Attribution License (CC BY). The use, distribution or reproduction in other forums is permitted, provided the original author(s) and the copyright owner(s) are credited and that the original publication in this journal is cited, in accordance with accepted academic practice. No use, distribution or reproduction is permitted which does not comply with these terms. 